\title{
A novel stress response pathway regulates rRNA biogenesis
}

Witold Szaflarski 1*, Mateusz Sowiński*, Marta Leśniczak1, Sandeep Ojha2,3, Anaïs Aulas4, Dhwani Dave5, Sulochan Malla2,3, Paul Anderson5,6, Pavel Ivanov5,6, Shawn M. Lyons2,3

1Department of Histology and Embryology, Poznan University of Medical Sciences, Poznań, Poland; 2Department of Biochemistry, Boston University School of Medicine, Boston, MA, USA; 3The Genome Science Institute, Boston University School of Medicine, Boston, MA, USA 4Predictive Oncology Laboratory, Cancer Research Center of Marseille (CRCM), Inserm U1068, CNRS UMR7258, Institut Paoli-Calmettes, Aix Marseille Université, Marseille, France; 5Division of Rheumatology and Clinical Immunology, Brigham and Women's Hospital, Boston, MA, USA; ${ }_{6}$ Department of Medicine, Harvard Medical School, Boston, MA, USA

${ }^{*}$ Co-First Authors 


\section{ABSTRACT}

2 Production of ribosomes is an energy-intensive process owing to the intricacy of these massive

3 macromolecular machines. Each human ribosome contains 80 ribosomal proteins and four non-coding

4 RNAs. Accurate assembly requires precise regulation of protein and RNA subunits. In response to stress,

5 the integrated stress response (ISR) rapidly inhibits global translation. How rRNA is coordinately

6 regulated with the rapid inhibition of ribosomal protein synthesis is not known. Here we show that stress

7 specifically inhibits the first step of rRNA processing. Unprocessed rRNA is stored within the nucleolus,

8 and, when stress resolves, it re-enters the ribosome biogenesis pathway. Retention of unprocessed rRNA

9 within the nucleolus aids in the maintenance of this organelle. This response is independent of the ISR or

10 inhibition of cellular translation but represents an independent stress-response pathway that we term

11 Ribosome Biogenesis Stress Response (RiBiSR). Failure to coordinately regulate ribosomal protein

12 translation and rRNA production results in nucleolar fragmentation. Our study unveils a novel stress

13 response pathway that aims at conserving energy, preserving the nucleolus, and prevents further stress

14 by regulation of rRNA processing.

15

16

17

18

19

20

21

22

23

24

25

26

27 


\section{INTRODUCTION}

31 Production of ribosomes is a major energetic and pro-growth task. Some estimates suggest that nearly $3260 \%$ of the cell's energetic costs are a result of ribosome production (Warner et al. 2001). The expenditure 33 of such a large percentage of energetic reserves is a result of the engagement of all three nuclear RNA 34 polymerases (Pol I: 18S, 28S, 5.8S rRNA, Pol II: ribosomal protein mRNA, Pol III: 5S rRNA), coordination 35 of ribosomal protein mRNA translation, modification of rRNAs and assembly in the nucleolus. Compounding these energetic demands is the requirement for the accurate processing of the $\sim 13,000$ nucleotide pre-

37 rRNA (47S rRNA) into mature 18S, 5.8S, and $28 \mathrm{~S}$ rRNAs. Again, estimates suggest that $60 \%$ of de novo RNA synthesis is rRNA. Maturation of the 47S rRNA requires multiple endonucleolytic and exonucleolytic processing events. The identity and processes that regulate some of these nucleases are yet to be identified. Further, these rRNAs are heavily chemically modified, making them second only to tRNAs in

41 terms of the percentage of modified nucleotides. The efficient assembly of ribosomes requires precise

42 regulation of rRNA transcription, processing, modification, and delivery of newly synthesized ribosomal proteins from the cytoplasm to the nucleolus.

45 Transcription and many of the steps in ribosome assembly takes place in a specialized membrane-less organelle known as the nucleolus. Maintenance of the nucleolar structure is driven, in part, by a liquid-liquid

47 phase separation (LLPS) (Brangwynne et al. 2009). Two main factors responsible for this process are RNA 48 and RNA-binding proteins that contain intrinsically disordered regions and low complexity sequences 49 (IDR/LCS). Two major IDR-containing proteins in the nucleolus are fibrillarin (FBL) and nucleophosmin 50 (NPM) (Feric et al. 2016; Mitrea et al. 2016; Yao et al. 2019). Additionally, electrostatic interactions target 51 proteins containing Nucleolar Localization Signals (NoLS) to the nucleolus (Martin et al. 2015). The 52 concentration of RNA processing and RNA modification enzymes in the nucleolus and other nuclear bodies

53 is thought to increase the efficiency of processing and modification (Dundr and Misteli 2010). Increases in 54 nucleolar size or changes in nucleolar morphology are linked to increased growth demands owing to the 55 necessity for new ribosomes in driving protein synthesis (Montanaro et al. 2008). 
57 The nucleolus has emerged as a significant stress-regulated organelle [Reviewed in (Boulon et al. 2010)].

58 In response to many stresses, such as heat shock (Liu et al. 1996), serum starvation (Chan et al. 1985),

59 nucleotide deprivation (Grummt and Grummt 1976), and UV irradiation (Zatsepina et al. 1989), the nucleolar

60 structure is fragmented and disrupted (Rubbi and Milner 2003). In most of these instances, nucleolar

61 disruption is a result of the inhibition of rRNA transcription. Nucleolar structure can be disrupted when levels

62 of newly synthesized RNA driving LLPS are lowered. In response to particular stresses (e.g., heat shock or

63 acidosis), RNAs are transcribed by RNA polymerase II between rDNA genes in regions known as intergenic

64 spacers (IGS), leading to the formation of reversible functional amyloids that may be cytoprotective (Audas

65 et al. 2012; Audas et al. 2016; Lyons and Anderson 2016).

67 Adverse environmental conditions activate cellular stress response pathways. Diverse exogenous stresses,

68 such as thermal stress, viral infection, oxidative stress or nutrient deprivation, elicit an equally diverse array

69 of cellular responses. However, the underlying goal of each of these responses is to promote survival. A

70 significant element of these pathways is to redirect energy from housekeeping and pro-growth activities

71 towards cell survival strategies. Upregulation of a subset of genes (e.g., ATF4) promotes survival during

72 stress. However, the expression of the vast majority of genes is downregulated, both transcriptionally and

73 translationally.

75 Our previous work has shown that acute stress causes global translation inhibition following elF2 $\alpha$

76 phosphorylation, which results in the formation of stress granules (SGs), non-membranous liquid-liquid

77 phase separations of untranslated mRNPs. The process by which this occurs is known as the "integrated

78 stress response (ISR)." The translation of mRNAs encoding ribosomal proteins is preferentially inhibited

79 and targeted to SGs upon elF2 $\alpha$ phosphorylation (Damgaard and Lykke-Andersen 2011). Additionally, the

80 translation of ribosomal protein mRNAs is a primary target of the second major stress-response pathway

81 that centers on the activity of mammalian target of rapamycin (mTOR) (Thoreen et al. 2012). Inactivation

82 of mTOR preferentially inhibits the translation of ribosomal protein mRNAs. Additionally, the transcription

83 of rRNA genes by RNA Pol I depends upon active mTOR (Mayer et al. 2004). Therefore, mTOR modulation

84 directly regulates both the translation of ribosomal protein mRNAs and the transcription of rRNA. This 
85 coordinate regulation is important because cells must balance rRNA synthesis with de novo ribosome

86 protein synthesis. Failure to do so results in nucleolar stress (Yang et al. 2018), which would only further

87 compound the initial cellular insult. While the translation of ribosomal protein mRNAs is similarly affected

88 by activation of the ISR, it is not known how this is coordinately regulated with rRNA biosynthesis. Further,

89 the ISR is activated under acute stress conditions (typically $<30 \mathrm{~min}$ ) and rapidly inhibits translation. Thus,

90 there is little leeway to regulate rRNA biosynthesis coordinately with the rapid shutoff of translation. We

91 hypothesize that failure in such coordination results in a misallocation of energetic resources leading to

92 further dysfunction.

94 Here, we show that stresses that induce elF2 $\alpha$ phosphorylation and SG formation also cause inhibition of 95 rRNA synthesis. Rather than directly inhibiting rRNA transcription by targeting the RNA polymerase I or associated basal transcription factors, leading to the disruption of the nucleolus, as has been shown for

97 mTOR-dependent stress responses, we show that the first step in 47S rRNA processing is inhibited. As the 98 rate of rRNA transcription is intrinsically tied to the efficiency of rRNA processing (Schneider et al. 2007), 99 failure to convert 47S rRNA into the next pre-RNA intermediate (45S rRNA) has the ultimate effect of 100 repressing the rate of rDNA transcription. Therefore, rRNA production is "paused" rather than being 101 inhibited. The unprocessed pre-rRNA is stored within the nucleolus until stress has resolved, at which point 102 it can re-enter the ribosome biogenesis pathway.

103 Moreover, this mechanism allows for the maintenance of nucleolar structure during stress as RNA is a 104 contributing factor in promoting LLPS in RNA granules (such as SGs or nucleolus). Retention of 105 unprocessed rRNA within the nucleolus during stress aids in the maintenance of nucleolar structure, such 106 that when stress has passed, ribosome biogenesis machinery remains localized to the nucleolus. In 107 contrast, direct inhibition of Pol I transcription compromises nucleolar integrity, thereby necessitating 108 reassembly of the nucleolus after stress has passed. Further, we show that despite being coordinated with 109 elF2 $\alpha$ phosphorylation, stress-responsive modulation of rRNA processing is regulated by an independent 110 yet parallel signaling pathway. Finally, we show that failure to regulate rRNA production coordinately with 111 translation results in nucleolar dysmorphology. Our data unveil a novel mechanism by which the first 
112 processing event in rRNA processing is regulated in a stress-dependent manner to conserve energetic

113 reserves and maintains nucleolar structure during stress.

\section{Results}

116 We began our investigation of the connection between rRNA biosynthesis and stress response by analyzing

117 the effect of stress on rRNA transcription using the 5-ethenyl uridine (5-EU) CLICK-IT assay. U2OS 118 osteosarcoma cells were stressed for 90 minutes with $\mathrm{NaAsO}_{2}$, the most widely used inducer of ISR, then 119 5-EU was added for an additional 30 minutes along with $\mathrm{NaAsO}_{2}$ (Figure 1A). Alternatively, cells were left 120 untreated or were treated with Actinomycin D (ActD), a potent inhibitor of transcription. Cells were fixed, 121 permeabilized, and Alexa488 fluorophore was conjugated to the incorporated 5-EU to visualize nascent 122 RNA. To mark the nucleolus, we used antibodies against NPM, a resident of the granular component (GC) 123 subcomponent of the nucleolus. In control conditions, a high concentration of nascent RNA is present in 124 the nucleolus consistent with rRNA accounting for the vast majority of transcription (Figure 1Ba). We did 125 not detect active transcription in the nucleolus after 90 minutes of $\mathrm{ActD}$ or $\mathrm{NaAsO}_{2}$ treatment by this assay 126 (Figure 1Bb - c). However, this presented a conundrum since nucleolar integrity was disrupted by ActD 127 but not $\mathrm{NaAsO}_{2}$. Therefore, we more directly interrogated rRNA by northern blotting. In humans, the primary 128 ribosomal RNA transcript, termed the 47S rRNA, is polycistronic, containing the 18S, 28S and 5.8S rRNA. 129 The mature rRNAs are flanked by 5' and 3' external transcribed spacers (5' and 3'ETS) and separated by 130 two internal transcribed spacers (ITS1 and ITS2). 47S rRNA is transcribed by RNA polymerase I and must 131 be reiteratively processed by multiple enzymes to release the mature rRNAs (Figure 1C). The 5S rRNA is 132 transcribed from other loci by RNA polymerase III. Using probes to different parts of the 47S transcript, we 133 can analyze different rRNA processing intermediates. We began by analyzing the full 47S rRNA with a 134 probe in the 5'ETS at the extreme 5' end of the rRNA. Our previous work, and those of others, has shown 135 that certain chemotherapeutic drugs are potent inducers of the ISR and a prevalent hypothesis has been 136 that disrupting ribosome biogenesis would be a potent strategy in combating tumor progression (Szaflarski 137 et al. 2016; Catez et al. 2019). Therefore, we expanded our analysis to include the chemotherapeutic drug 138 lomustine. Similar to $\mathrm{NaAsO}_{2}$, lomustine induces phosphorylation of elF2 $\alpha$, the hallmark of ISR induction, 139 while ActD does not (Figure S1A). However, both $\mathrm{NaAsO}_{2}$ and Lomustine treatments resulted in a striking 
increase in 47S precursor rRNA and the formation of a faster migrating species (Figure 1D, In 3, 4). The changes in 47S expression were independently confirmed by qRT-PCR (Figure 1E). The faster migrating

142 species that has alternatively been termed the $30 S_{+01}$ fragment or the $34 S$ fragment is often seen when

143 small subunit (SSU) processome is inhibited, e.g., under RNAi mediated knockdown of certain RNA 144 processing factors such as fibrillarin (Tafforeau et al. 2013). This fragment results from a failure to process 145 the first cleavage site, the A'/01 site (Figure 1C), and spurious processing at the "site 2" site. Further 146 northern blotting for ITS1 and ITS2 revealed a decrease in downstream processing intermediates (e.g. 41S, $14726 S, 21 S$, 18S-E and 12S) (Figure 1D and S1B - E). It is worth noting that northern blotting to ITS1 does 148 not distinguish between the aberrant 34S product and canonical 30 S precursors. Regardless, these data 149 suggest that pre-rRNA processing is inhibited by preventing $A^{\prime} / 01$ processing, leading to an increase in 47S 150 pre-rRNA and a decrease in downstream precursors.

151 The transcription factor p53 plays a major role in the maintenance of the nucleolus (Rubbi and Milner 2003; 152 Woods et al. 2015). U2OS cells are a p53 positive cell line, so, to address the possibility that regulation of 153 rRNA processing was dependent upon p53, we performed the same experiments in HeLa cells, which are 154 p53-null due to overexpression of the Human Papillomavirus E6 gene (Liu et al. 1999). We found that 155 regulation of processing was independent of p53 status as it occurs in both the p53-positive U2OS cells 156 and the p53-deficient HeLa cells. We also wanted to address whether or not this regulation was specific to 157 cancer cells, so we assayed non-cancerous human RPE-1 cells that had been immortalized with hTERT. 158 Again, we found the regulation as in the cancerous U2OS and HeLa cells (Figure S1C). Finally, the A'/01 159 processing site is conserved in mice, so we wanted to assay if the regulation of processing was also 160 conserved across evolution. Using NIH3T3 cells, we found the same increase in 47S rRNA, loss of 161 downstream precursors and generation of 34S pre-rRNA intermediate (Figure S1D). Finally, we show that 162 loss of downstream precursors and generation of the 34S fragment occurs in a dose-dependent and time163 dependent manner (Figure S1E). Therefore, stress-dependent regulation of rRNA processing is an 164 evolutionarily conserved mode of stress response that occurs in response to various stresses, in a p53165 independent manner in both transformed and untransformed cells. 
167 The rate and efficiency of RNA processing strongly affect the rate of transcription and vice-a-versa 168 (Schneider et al. 2007). That is, inhibition of rRNA processing can feedback to reduce rRNA transcription.

169 The accumulation of $47 \mathrm{~S}$ rRNA coupled with the loss of transcription after 90 minutes of stress suggested 170 that rRNA processing was inhibited, resulting in accumulation of the primary transcript (Figure 1 D $-\mathbf{E}$ ), 171 which eventually results in cessation of RNA transcription (Figure 1A - 1B). Thus, we complemented our 172 analysis of processing by ${ }_{22} \mathrm{P}-$ metabolic labeling (Figure $2 \mathrm{~A}-\mathbf{C}$ ). Here, cells were stressed and [32P]-ortho173 phosphoric acid was added to monitor rRNA transcription and processing before chasing with cold, $174 \quad \mathrm{NaAsO}_{2}$-containing media for 2.5 hours (Figure 2A) in accordance with published protocols (Pestov et al. 175 2008). In control conditions, initial precursors (47S, 45S), intermediates (30, 32S) and mature rRNAs (28S, 176 18S) were all observed and matured over the 3.5-hour time-course (Figure 2B, In 15 - 21). However, in 177 agreement with northern blotting data, after $\mathrm{NaAsO}_{2}$ treatment, initial precursors were generated, but these 178 never matured to 18 S and 28S rRNAs (Figure 2B, In 22 - 28). We did observe RNA species approximately 179 the size of the 30S and 32S precursors that our northern blotting data, particularly our dose-response data 180 (Figure S1E), would suggest that it is the 34S fragment, and not a canonical processing intermediate. 181 However, this assay does not allow us to identify this fragment unambiguously. Regardless, these data 182 confirm that rRNA processing is inhibited by stress rather than directly targeting transcription. Treatment 183 with the transcriptional inhibitor, Actinomycin D, completely abolished new synthesis of rRNA (Figure 2C). 184 We propose that inhibition of pre-rRNA processing serves to "pause" ribosome biogenesis during a stress 185 response. This occurs coordinately with inhibition of ribosomal protein biogenesis caused by elF2 $\alpha$ 186 phosphorylation. However, for this "pausing" to be an effective stress response strategy, the accumulated 187 47S rRNA should be utilized when stress has passed. Upon $\mathrm{NaAsO}_{2}$ washout, elF2 $\alpha$ is rapidly 188 dephosphorylated and translation resumes (Novoa et al. 2003). To assay if this occurs, we performed the 189 same ${ }_{32} \mathrm{P}$-metabolic labeling experiments, but followed the labeled $\mathrm{RNA}$ after removing $\mathrm{NaAsO}_{2}$. The timing 190 of this experiment is critical for accurate interpretation (Figure 2E). Cells were stressed with $\mathrm{NaAsO}_{2}$ 191 concurrently with ${ }_{32} \mathrm{P}$ labeling as before. ${ }_{32} \mathrm{P}$ was removed and excess cold phosphate was added. Finally, $192 \mathrm{NaAsO}_{2}$ was removed and we monitored rRNA biogenesis over a 9-hour time course. This experimental 193 setup demonstrated that rRNA was matured under control conditions as $18 \mathrm{~S}$ and $28 \mathrm{~S}$ rRAs were 194 generated by 2 hours. This process was stalled by $\mathrm{NaAsO}_{2}$ as demonstrated previously, but after washout, 
195 the radiolabeled pre-rRNA began to accumulate as mature rRNAs (Figure 2E). The final amount of pre-

196 rRNA that matured to $18 \mathrm{~S}$ and $28 \mathrm{~S}$ was significantly lower than under control conditions because of the

197 stalling in rRNA synthesis during stress. Therefore, during a stress response, rRNA processing stalls upon

198 inhibition of processing at the A'/01 processing site, leading to the accumulation of $47 \mathrm{~S}$ pre-rRNA. When

199 stress resolves, this stored 47S pre-rRNA re-enters the biogenesis pathway leading to the production of

200 mature rRNAs.

201

202 The transcription and processing of rRNAs and the maturation of ribosomes occur in the nucleolus. Since

203 this pathway is regulated in response to stress, we sought to investigate further if stress triggered any

204 changes to the nucleolus. While untransformed cells typically have $2-3$ nucleoli, it has been long

205 established that cancer cells often exhibit an increased number of nucleoli that are often of increased size,

206 an observation that has been shown to be correlated with proliferative potential (Montanaro et al. 2008).

207 Indeed, human osteosarcoma U2OS cells examined in this study have between 3 and 5 nucleoli on average

208 as monitored by immunostaining against NPM (Figure 3Aa \& 3B). Treatment of these cells with ActD,

209 significantly reduces the size or abolished the nucleoli as monitored by NPM (Figure $\mathbf{3 A b} \& \mathbf{3 C}$ ) without

210 affecting the nucleolar number of cells that retained nucleoli on a per-cell basis (Figure 3B). RNA and RNA-

211 binding proteins (RBPs) are major drivers of LLPS in non-membranous organelles (Lin et al. 2015; Protter

212 et al. 2018; Van Treeck et al. 2018). Thus, as transcription inhibition reduces RNA levels in the nucleolus,

213 nucleolar structure is disrupted. However, as previously shown, this is not coincident with activation of the

214 ISR (Figure S1A), which is further exemplified here by lack of stress granule formation as monitored by

215 elF3B localization (Figure 3A). Upon treatment with $\mathrm{NaAsO}_{2}$ or lomustine we induced the formation of

216 stress granules; however, NPM-positive nucleoli remained (Figure 3Ac-d). NPM is but one marker of

217 nucleoli which mass spectrometry analysis has determined contains over 270 protein (Andersen et al.

218 2002). These proteins are distributed throughout three subcompartments within the nucleoli: Granular

219 component (GC), Dense Fibrillar component (DFC) and Fibrillar component (FC). To ensure that NPM, a

220 GC resident protein, was not unique in its nucleolar retention, we analyzed 11 additional nucleolar

221 components by immunofluorescence representing members of each subcompartment and found no

222 appreciable change in localization; however, future research will be needed to analyze more subtle changes 
223 in nucleolar substructures. (Figure S2A - H). As with regulation of rRNA processing, using HeLa cells, we

224 also show that persistence of the nucleolus during stress is independent of p53 (Figure S2J).

226 It is worth noting that these data seemingly contradict a previous report that showed that oxidative stress

227 initiated by $\mathrm{H}_{2} \mathrm{O}_{2}$ inhibits rRNA synthesis via phosphorylation of TIF-1A, a basal polymerase I transcription

228 factor (Mayer et al. 2005). Thus, we sought to address this apparent discrepancy. In doing so, we repeated 229 previously reported data by showing that $\mathrm{H}_{2} \mathrm{O}_{2}$-induced oxidative stress disrupts nucleolar architecture

230 (Figure 3Ae, 3B - D). However, our previous data showed that $\mathrm{H}_{2} \mathrm{O}_{2}$ not only activates the ISR, but also 231 potently inhibits mTOR (Emara et al. 2012) as indicated by an increase in non-phosphorylated 4EBP1

232 (Figure S1A). Since mTOR is required for TIF-1A activation, the different targets of $\mathrm{H}_{2} \mathrm{O}_{2}-$ and $\mathrm{NaAsO}_{2}-$ 233 induced oxidative stress explains the apparent contradiction (Mayer et al. 2004). We conclude that 234 persistence of the nucleolus during stress is a feature of ISR-activating oxidative stress when mTOR is 235 active, but not when it is inactive.

237 To further analyze nucleolar size and morphology, we employed Imaris imaging software to reconstruct 3D 238 models of nucleolar structure under various cellular conditions to make parametrical analysis of the 239 organelles and, in consequence, more precise measurements (Figure 3C). This analysis revealed that 240 upon the initiation of a stress response, there was no apparent change in nucleolar volume, in contrast to 241 inhibition of transcription by ActD (Figure 3D). Immunofluorescence also suggested a change in nucleolar 242 morphology upon induction of a stress response, namely adoption of more rounded morphology. We 243 specifically quantified this by measuring sphericity as a ratio between the radius of an inscribing and 244 circumscribing circle of the nucleoli. Thus, the more spherical the nucleoli, the closer the sphericity will be 245 to 1 , and the more oblong, the closer the shape will be to 0 . Upon analysis, we showed a statistically 246 significant increase in sphericity after $\mathrm{NaAsO}_{2}$ and lomustine treatment, suggesting a change in the 247 biophysical dynamics of this organelle (Figure 3E).

249 Since RNA is a contributing factor of nucleolar assembly via an RNA-driven liquid-liquid phase separation, 250 we next sought to determine if this unprocessed rRNA was retained within the nucleolus, as would be 
251 suggested by the persistence of nucleoli and the utilization of stored pre-rRNA after stress (Figure 2 \& 3).

252 We performed RNA FISH in conjunction with immunofluorescence to analyze the localization of rRNA using

253 Imaris 3D reconstruction. Following stress, 47S rRNA was retained within the nucleolus which aids in the

254 preservation of nucleolar structure (Figure 4A). Maintenance of this RNA in the nucleolus (Figure 4 A),

255 inhibition of processing (Figure $1-2$ ) and increased nucleolar sphericity (Figure 3E) would suggest that

256 the unprocessed RNA is being stored in the nucleolus thereby effecting nucleolar dynamics during stress.

257 To assess this, we performed fluorescence recovery after photobleaching (FRAP) of nucleoli under

258 stressed and unstressed conditions. Cell lines stably expressing mCherry-Nol9, an rRNA processing factor,

259 GFP-RPL7A, a ribosomal protein, and mCherry-NPM were generated (Figure S3A). We performed FRAP

260 on control cells or cells treated with $\mathrm{NaAsO}_{2}$ for 2 hours (Figure 4B - E, Figure S3B - C). Since the

261 nucleolus is an extremely active organelle, we were not surprised to find that fluorescence recovered nearly

262 completely 45 seconds post-bleaching for each of the three tagged proteins, with NPM recovering after only

$26310-12$ seconds post-bleach. However, $\mathrm{NaAsO}_{2}$ treatment severely diminished nucleolar dynamics. We

264 observed almost no recovery over the same timescale. Particularly striking was the change in recovery of

265 Nol9, an rRNA processing factor. These data confirm that in response to stress, the nucleoli serve as

266 storage sites for unprocessed rRNA. The presence of this rRNA aids in the persistence of nucleoli.

267 We next sought to determine if regulation of rRNA processing in response to stress is a component

268 of the ISR. To begin, we analyzed transcription two hours post-stress using 5-EU as before (Figure 1A).

269 Formation of stress granules is typically seen as a proxy for ISR activation (Kedersha et al. 2013). Thus,

270 we treated cells with levels of $\mathrm{NaAsO}_{2}$ below the level that fully induces SG formation (Figure 5A). At 75

$271 \mu \mathrm{M} \mathrm{NaAsO}_{2}$, approximately $50 \%$ of cells have visible SGs as monitored by elF3B staining. However,

272 regardless of whether or not a cell has SGs or not, nucleolar transcription has ceased, suggesting that

273 regulation of rRNA is independent of the ISR. To further explore whether activation of ISR is a requirement

274 of stress-dependent rRNA regulation, we knocked out heme-regulated inhibitor kinase (HRI/EIF2AK1)

275 using CRISPR/Cas9 (Figure S4). We have previously shown that CRISPR/Cas9 knockout of HRI renders

276 cells unresponsive to $\mathrm{NaAsO}_{2}$ with regards to elF2 $\alpha$ phosphorylation, translational repression and SG

277 formation (Aulas et al. 2017). Therefore, we sought to determine whether rRNA processing was still

278 regulated if the ISR was inhibited by deletion of HRI. SGs form in wild-type U2OS cells in response to 
$279 \mathrm{NaAsO}_{2}$, while $\triangle \mathrm{HRI}$ U2OS cells fail to form SGs (Figure 5B). However, 5-EU labeling of nascent RNA 280 reveals transcriptional shutoff of nucleolar RNA synthesis two hours post-stress in both WT and $\Delta \mathrm{HRI}$ cells.

281 To further explore the connection, or lack thereof, between the ISR and rRNA regulation, we processed WT

282 and $\Delta \mathrm{HRI}$ cells for northern blotting analysis before and after stress. We still found the generation of the

$28334 \mathrm{~S}$ fragment after $\mathrm{NaAsO}_{2}$ treatment (Figure 5C and D) and a decrease in downstream rRNA precursors,

284 indicating that inhibition of pre-rRNA processing occurs in a stress-dependent manner, independent of the

285 ISR. Finally, we completed ${ }_{32} \mathrm{P}$ pulse-chase experiments to monitor rRNA processing. We found that, as in

286 WT U2OS cells, 47S rRNA was generated in $\triangle \mathrm{HRI}$ U2OS cells, but this never matured to $28 \mathrm{~S}$ or 18S rRNA

287 after induction of a stress response (Figure 5D). Therefore, regulation of rRNA biogenesis in response to

288 stress is a parallel, but independent, pathway to the ISR. We term this previously undescribed pathway the

289 "Ribosome Biogenesis Stress Response (RiBiSR)."

We have argued that RiBiSR serves to maintain cellular homeostasis during stress by preventing

291 unnecessary production of rRNA when ribosomal protein synthesis has been inhibited in the cytoplasm.

292 Further, we argue that it functions to maintain the balance between ribosomal protein synthesis and rRNA

293 synthesis, thereby preserving nucleolar integrity and protecting against nucleolar stress. To test this

294 hypothesis and understand what the consequences of disrupting this balance are, we treated cells with

295 puromycin (Puro) or cycloheximide $(\mathrm{CHX})$. Both Puro and $\mathrm{CHX}$ are pharmacologic inhibitors of translation

296 that do not activate the ISR or inactivate mTOR (Figure S1A). They both directly target the translational

297 machinery: Puro triggers premature translation termination while $\mathrm{CHX}$ stalls ribosome elongation.

298 Therefore, treatment with these drugs would inhibit de novo ribosomal protein synthesis without directly

299 targeting rRNA synthesis. Upon treatment of cells with $\mathrm{CHX}$ and Puro, despite inhibiting translation through

300 different mechanisms, we found similar results. We began by analyzing nucleolar dynamics by FRAP

301 (Figure 6A - D, Figure S5A - B). We argued that stress-induced decline in nucleolar dynamics served to

302 maintain nucleolar structure after the inhibition of ribosomal protein synthesis. However, upon translation

303 inhibition by Puro or $\mathrm{CHX}$, we found no significant change in nucleolar dynamics irrespective of the fact that

304 new ribosomal proteins are not being delivered to the nucleolus (judging by either RPL7A (Figure 6A - B)

305 or Nol9 (Figure 6C - D)). Next, we analyzed rRNA by northern blotting and found that 47S rRNA was still

306 present without the production of the stress-dependent 34S fragment (Figure 6E). However, there was a 
reduction in the initial 47S rRNA precursor (Figure 6E, In 9 \& 10). Additionally, downstream precursors (e.g., 12S, 21S, 18S-E) are reduced upon treatment with Puromycin and $\mathrm{CHX}$, consistent with the reduction of $47 \mathrm{~S}$, but not abolished as it is seen with $\mathrm{NaAsO}_{2}$. This is consistent with cells maintaining the balance between ribosomal protein and rRNA synthesis. However, pharmacological inhibition of ribosomal protein synthesis results in an inhibition of $47 \mathrm{~S}$ synthesis, not processing. Finally, we have argued that stressdependent regulation of rRNA processing preserves nucleolar integrity when ribosomal protein synthesis has been inhibited. As $\mathrm{CHX}$ and puromycin do not induce regulated inhibition of rRNA processing, we sought to determine the effect of continued rRNA production in the absence of ribosomal protein synthesis

315 on nucleolar morphology by immunofluorescence (Figure 6F). As we found previously, nucleolar structure 316 was preserved after $\mathrm{NaAsO}_{2}$-induced stress, with individual nucleoli adopting a more spherical morphology,

317 concurrent with the formation of stress granules in the cytoplasm (Figure 6Fb). In contrast, neither $\mathrm{CHX}$ 318 nor puro induces SG formation, but there was a dramatic effect on nucleolar morphology (Figure 6Fc-d). 319 Intact nucleoli adopted a "ragged" morphology. However, more striking, was that the nucleoli in a subset of cells became fragmented indicating a total loss of nucleolar integrity. The fact that there was no

321 compensatory nucleolar response to the loss of ribosomal protein production likely contributes to the 322 resultant nucleolar fragmentation. These results demonstrate the importance of RiBiSR for maintenance of 323 cellular homeostasis under stress.

\section{DISCUSSION}

327 Our work reveals a novel stress-response pathway in mammalian cells that we have termed RiBiSR. This 328 pathway is independent of the ISR, which functions in the cytoplasm to initially inhibit translation initiation.

329 This novel program regulates the biogenesis of ribosomes, amongst the most energy-intensive, pro-

330 growth processes in the cell. The goal of any stress response is to promote survival until the return to 331 homeostasis. This is largely accomplished by the reallocation of energy reserves away from pro-growth 332 activities towards pro-survival activities. Since the majority of cellular energy is devoted to ribosome 333 biogenesis (Warner et al. 2001), it is unsurprising that this pathway is under substantial regulatory 334 pressure during a stress response. Much work has gone into demonstrating how protein synthesis and 
335 specifically the translation of ribosomal proteins is regulated in response to stress (Ivanov et al. 2011; Meyuhas and Kahan 2015). Others have previously shown that inactivation of mTOR in response to

337 nutrient starvation, downregulates the transcriptional rate of rRNA genes (Mayer et al. 2004). Additionally,

338 in yeast, TORC1 signaling has also been implicating in regulating the processing of rRNA in response to 339 certain stresses (Kos-Braun et al. 2017). Here, we uncover a novel mechanism that is responsive to 340 acute stress that functions via regulation of rRNA processing. Our data show that upon activation of the

341 ISR, the nucleolus is maintained by inhibiting rRNA processing rather than inhibiting rRNA transcription.

342 The initial processing event, conversion of the $47 \mathrm{~S}$ to $45 \mathrm{~S}$ rRNA, is under tight stress-dependent

343 regulation. Unlike other stress response pathways that regulate ribosome biogenesis, this pathway is not

344 dependent upon mTOR, nor is it dependent upon ISR-mediated translation repression.

346 Importantly, this mechanism of regulation results in the preservation of the nucleolus. A major contributing

347 factor to nucleolar structure is driven by a liquid-liquid phase separation (LLPS). FBL, the 2'O

348 methyltransferase, aids in the establishment of an LLPS by binding nascent rRNAs and sorting them into 349 nucleolar compartments (Yao et al. 2019). Additionally, the multimerization of NPM plays a critical role in 350 establishing nucleolar structure (Mitrea et al. 2018). Since RNA is a major driver of LLPS [Reviewed in 351 (Van Treeck and Parker 2018)], stresses or cellular conditions that inhibit rRNA transcription result in the 352 dissolution of nucleolar structure due to the absence of rRNA in this compartment (ActD, Figure 3).

353 Nuclear bodies, including the nucleolus, are often formed around regions of high transcriptional output 354 and serve to concentrate factors involved in RNA metabolism (Reviewed in (Dundr and Misteli 2010)). In 355 addition to the nucleolus, this has been shown for other nuclear bodies, including the histone locus body 356 (Tatomer et al. 2016) and Cajal bodies (Xu et al. 2005). Therefore, if a stress response were to result in 357 the destruction of the nucleolus, this would only further hamper cell viability as the cell recovers from a 358 cellular insult. Nucleolar components would be dispersed throughout the nucleoplasm and need to be 359 relocalized to begin ribosome biogenesis. We propose that by maintaining nucleolar structure, an efficient 360 return to homeostatic conditions is ensured upon the cessation of cellular insult without wasting an 361 additional energy in reassembling this nuclear body. 
363 We propose that this mechanism has parallels to the role of translation inhibition following activation of 364 the ISR that results in phosphorylation of elF2 $\alpha$ by one of four stress-responsive kinases. This pathway 365 culminates in the stalling of translation at the initiation stage, but, importantly, this does not result in the 366 degradation of mRNAs or the disassembly of translation initiation complexes. Instead, stalled 48S pre-

367 initiation complexes and mRNAs are stored in SGs, which themselves are liquid-liquid phase condensates. As the stressed state resolves, stored mRNAs can re-enter the pool of translating mRNAs.

369 Stress-dependent regulation of rRNA processing does not destroy rRNA, but stalls its biogenesis at the 370 initial stages, similar to inhibition of translation initiation. Additionally, regulation of processing preserves 371 the localization of ribosome biogenesis machinery within the nucleolus. This is in contrast to mTOR372 inactivating stresses that affect both rRNA transcription and mRNA translation. Starvation conditions are 373 particularly potent inhibitors of mTOR. With regards to translation, this results in the dephosphorylation of 374 4EBP1 proteins which disrupt the elF4F and pre-initiation complex formation. Similarly, mTOR

375 inactivation results in inhibition of transcription of rDNA genes through regulation of TIF-1A/RRN3 (Mayer 376 et al. 2004). Here, this results in disassembly of the nucleolus which disperses rRNA processing enzymes 377 throughout the nucleoplasm. In yeast, TORC1 signaling has been shown to affect the processing of the 378 35S rRNA by causing a switch in the site of ITS1 processing (Kos-Braun et al. 2017).

Our data also suggests that conversion of the $47 \mathrm{~S}$ to $45 \mathrm{~S}$ rRNA is an obligatory step in rRNA biogenesis.

381 When the pre-RNA matures to the 45S intermediate, there are multiple pathways through which the RNA 382 intermediate can mature (reviewed in (Mullineux and Lafontaine 2012)). However, our results 383 demonstrate that, when stalled as the 47S rRNA, there is no alternative pathway that leads to mature $38418 \mathrm{~S}, 5.8 \mathrm{~S}$ and $28 \mathrm{~S}$ rRNAs. The fraction of $47 \mathrm{~S}$ that gets processed to the $34 \mathrm{~S}$ is not matured despite the 385 fact that this represents the same RNA species as 30S rRNA except for the 5' extension (i.e. 1 - 01 386 fragment). It is tempting to speculate that this region contains an inhibitory sequence that prevents further 387 processing. Alternatively, all rRNA processing enzymes could be simultaneously inhibited, but this would 388 necessitate a here-to-fore unforeseen level of rRNA processing regulation. Another outstanding question 389 is whether the 34S rRNA has any biological function. It is generated in a dose- and time-dependent manner during a stress response (Figure S1E). This rRNA species has been observed upon the 
391 knockdown of rRNA processing components necessary for small subunit biogenesis (e.g. FBL) (Tafforeau

392 et al. 2013). Our FISH data demonstrates that this fragment is retained in the nucleolus, but whether it

393 plays a functional or structural role there is unknown.

394

395 Importantly, the enzyme responsible for cleavage at the $A^{\prime} / 01$ site in the $5^{\prime}$ ETS is unknown. This initial

396 processing site is conserved in mice and humans (Mullineux and Lafontaine 2012), as is the stress-

397 dependent regulation (Figure S1). Processing here, and at the 02 site at the 3 ' end of the pre-rRNA,

398 convert the 47S rRNA to the 45S rRNA. The identity of this enzyme (or enzymes) and how its activity is

399 regulated in response to stress are critical to gain a full understanding of the cellular stress response. We

400 show that this pathway is parallel to, but not dependent upon ISR activation (Figure 5). This suggests the

401 existence of an alternative stress-responsive pathway found in the nucleus or nucleolus. However, the

402 identity of the players in this pathway remain to be uncovered.

403

404

MATERIALS AND METHODS

405

406

Antibodies

407 TIAR (Santa Cruz, sc-1749), NPM (Santa Cruz, sc-70392), FBL (Cell signaling Technology, 2639),

408 RPA194 (Santa Cruz, sc-4669), elF2 $\alpha$ (Santa Cruz, sc-133132), phospho-elF2 $\alpha$ (AbCam, 131505), 4EBP

409 (Cell Signaling Technology, 9454), non-phospho-4EBP (Cell Signaling Technology, 4923S), TIF-

410 1A/RRN3 (Santa Cruz, 2c-390464), RPL7A (Cell Signaling Technology, 2415) Nol9 (Protein Tech Group,

411 16083-1-AP) , UBF (Santa Cruz, sc-13125), elF3B (Santa Cruz, sc-16377)

\section{Cell culture and drug treatment}

414 U2OS and HeLa cells were maintained in DMEM supplemented with 10\% Fetal Bovine Serum and

415 Penicillin/Streptomycin. NIH3T3 were maintained in DMEM supplemented with 10\% Bovine Calf Serum

416 and Penicillin/streptomycin in a humidified $37 \circ \mathrm{C} / 5 \% \mathrm{CO}_{2}$ incubator. $\mathrm{NaAsO}_{2}$ (Sigma), Lomustine

417 (Selleckchem), $\mathrm{H}_{2} \mathrm{O}_{2}$ (Fisher), Actinomycin D (Arcos Organics) were added for indicated times at

418 indicated concentration. Where not noted, $\mathrm{NaAsO}_{2}$ was treated at $200 \mu \mathrm{M}$. 


\section{Epifluorescence Immunofluorescence}

421 Cells were fixed and processed for fluorescence microscopy as described (Lyons et al. 2016). Briefly,

422 cells were grown on glass coverslips, stressed as indicated and fixed with $4 \%$ paraformaldehyde in PBS

423 for 15 minutes followed by 10 minutes post-fixation/permeabilization in -20 。C methanol. Cells were

424 blocked for 1 hour in 5\% horse serum/PBS. Primary and secondary antibody incubations were performed

425 in blocking buffer for 1 hour with rocking at room temperature. Secondary antibodies (Jackson

426 Laboratories) were tagged with Cy2, Сy3 or Cy5. Following washes with PBS, cells were mounted in

427 polyvinyl mounting media and viewed at room temperature using a Nikon Eclipse E800 microscope with a

$42840 X$ Plan fluor (NA 0.75) or 100X Plan Apo objective lens (NA 1.4) and illuminated with a mercury lamp

429 and standard filters for DAPI (UV-2A -360/40; 420/LP), Cy2 (FITC HQ 480/40; 535/50), Cy3 (Cy 3HQ

430 545/30; 610/75), and Cy5 (Cy 5 HQ 620/60; 700/75). Images were captured with SPOT Persuit digital

431 camera (Diagnostic Instruments) with the manufacturers software and compiled using Adobe Photoshop

4322020.

\section{Imaris 3D reconstruction and Parametric analysis}

435 For Imaris imaging, cells were treated in the same way as for epifluorescence immunofluorescence. The 436 crude Z-stack images were collected using Olympus FV10i confocal laser scanning microscope. Then, 437 images were processed with Imaris 7.4.2 (Bitplane, UK) in order to get 3D reconstruction. The sizes of 438 nuclei and nuclei were automatically calculated on the basis of Z-stacks composed of individual images. 439 We then calculated the volume of all nucleoli in one nucleus in one cell. At least 100 cells from at least 440 three independent experiments were taken for one analysis.

\section{Metabolic labeling}

443 For 5-ethynyl uridine labeling experiments, experiments were conducted using Click-iT RNA Alexa Flor 444488 Imaging Kit (ThermoFisher) according to manufacturer's instructions. Cells were prepared as for 445 immunofluorescence, but 30 minutes prior to fixation, 5-EU was added to a final concentration of $1 \mathrm{mM}$. 446 For $[32 \mathrm{P}]-$-metabolic labeling, cells were grown in a 6-well dish. Cells were starved for one hour in 
447 phosphate free DMEM containing 10\% dialyzed FBS. Cells were then treated for 10 minutes with

448 indicated compounds before addition of $20 \mu \mathrm{Ci}$ of ${ }_{32} \mathrm{P}$-ortho- phosphoric acid (Perkin Elmer). Cells were

449 incubated for 1 hour with media containing drugs and $32 \mathrm{P}$-ortho- phosphoric acid, before replacing with

450 DMEM containing 10\% FBS with indicated drug treatment for an additional 1.5 hours. RNA was harvested

451 with Trizol reagent according to manufacturer's instructions and resuspended in $30 \mu$ l of RNA loading

452 dye. Ten microliters of RNA was loaded onto a 1.2\% Agarose gel made with 1X H-E buffer (20 mM

453 HEPES, $1 \mathrm{mM}$ EDTA [pH 7.8] and 7\% Formaldehyde. Gels were run overnight in 1X H-E buffer at $55 \mathrm{~V}$

454 with recirculation and then dried and exposed to film.

$456 \quad$ Northern Blotting

457 Cells were grown to $\sim 80 \%$ confluency and RNA was extracted with Trizol reagent (Invitrogen) according

458 to manufacturer's instructions. $5 \mu \mathrm{g}$ of extracted RNA was resuspended in RNA loading dye ( $7 \mu$ l of

459 Formamide, $2 \mu$ l of Formaldehyde, $1 \mu \mathrm{L}$ of 10X HEPES-EDTA Buffer, $1 \mu$ l of Ethidium bromide $(0.4$

$460 \mathrm{mg} / \mathrm{ml}), 1 \mu \mathrm{l}$ of bromophenol blue $(0.5 \mathrm{mg} / \mathrm{ml})$ and heated to 85 。C for 10 minutes before placing on ice.

461 Denatured RNA was loaded onto a 1.2\% Agarose gel prepared in H-E buffer (20 mM HEPES, 1 mM

462 ETDA [pH 7.8] and 7\% formaldehyde. Gel was run in HEPES-EDTA buffer overnight at 55V with

463 recirculation. The following day, the gel was subjected to mild alkaline treatment (10 minutes in $50 \mathrm{mM}$

$464 \mathrm{NaOH} / 10 \mathrm{mM} \mathrm{NaCl}$ ), neutralization (10 minutes in 2.5X TBE) and equilibration in 2X SSC. RNA was

465 transferred overnight by passive transfer to Hybond $\mathrm{N}_{+}$Nylon membrane using 20X SSC. The following

466 day, RNA was dried, crosslinked to membrane and pre-hybridized in $10 \mathrm{ml}$ of UltraHyb pre-

467 hybridization/hybridization solution (Invitrogen) for 1 hour at 60॰C. Pre-hybridization solution was removed

468 and $10 \mathrm{~mL}$ of fresh UltraHyb was added along with $15 \mu \mathrm{l}$ of end labeled northern probe. Probe was

469 incubated for 1 hour at $60 \circ \mathrm{C}$ and then overnight at $37 \circ \mathrm{C}$. The following day, probes were washed twice

470 with $2 \times$ SSC/0.1\% SDS at 40。C and exposed to film. For stripping and re-probing, $50 \mathrm{~mL}$ of boiling $0.1 \mathrm{X}$

471 SSC/0.1\% SDS was added to blots and allowed to come to room temperature twice.

472

\section{Preparation of northern blotting probes}


474 Synthetic DNA oligonucleotides were prepared by Integrated DNA Technologies and resuspended to a

475 final concentration of $6 \mu \mathrm{M}$ in $\mathrm{dH}_{2} \mathrm{O}$. For end-labeling, $1 \mu \mathrm{l}$ of DNA was reacted with $2 \mu \mathrm{l}$ of [32P]- $\gamma-\mathrm{ATP}$

$476(3000 \mathrm{Ci} / \mathrm{mL})$ (Perkin-Elmer), $1 \mu \mathrm{l}$ of $10 \mathrm{X}$ T4 PNK buffer, $1 \mu \mathrm{l}$ of T4 PNK (NEB), and $14 \mu \mathrm{l}$ of $\mathrm{dH} \mathrm{H}_{2} \mathrm{O}$ for 1

477 hour. The reaction was brought to $100 \mu \mathrm{l}$ with $\mathrm{dH}_{2} \mathrm{O}$ and unincorporated nucleotides were removed by gel

478 filtration through G-25 column (GE LifeSciences). The following oligonucleotide sequences were used for

479 northern blotting: Human 5' ETS

480 (CGGAGGCCCAACCTCTCCGACGACAGGTCGCCAGAGGACAGCGTGTCAGC), Human ITS1

481 (GGCCTCGCCCTCCGGGCTCCGTTAATGAT), Human ITS2 (CTGCGAGGGAACCCCCAGCCGCGCA),

482 Mouse 5’ETS (AAGCAGGAAGCGTGGCTCGGGGAGAGCTTCAGGCACCGCGACAGA), Mouse ITS1

483 (ACGCCGCCGCTCCTCCACAGTCTCCCGTTTAATGATCC), Mouse ITS2

484 (ACCCACCGCAGCGGGTGACGCGATTGATCG).

\section{qRT-PCR}

487 For qRT-PCR cells were growing on 6-well plate. After treatment with corresponding drug, total RNA was

488 isolated using Universal RNA/miRNA Purification Kit (EURx, Poland). RNA was quantified using

489 Nanodrop ND-1000 (ThermoScientific, USA). cDNA was obtained using LunaScript ${ }^{\mathrm{TM}}$ RT SuperMix Kit

490 (New England Biolabs, USA) and qPCR reactions were performed using Luna® Universal qPCR Master

491 Mix (New England Biolabs, USA) according to manufactural instructions. The qPCR reactions were done

492 in CFX96 Touch Real-Time PCR Detection System (Bio-Rad, USA) and then analyzed in CFX Maestro

493 Analysis Software (Bio-Rad, USA). The final graphs were prepared in GraphPad Prism 8. Primers for

494 amplification spanned the A'/01 site ensuring that only 47S and not other precursors were amplified. The

495 sequences were: GTGCGTGTCAGGCGTTC and GGGAGAGGAGCAGACGAG.

\section{FRAP}

498 U2OS cells were transfected with plasmids containing tagged versions of RPL7A, Nol9 or NPM and 499 selected with geneticin. Cells were grown in 4-compartment $35 \mathrm{~mm}$ glass bottom dish (Greiner) until $500 \sim 80 \%$ confluency. Cells were treated with indicated drugs for 2 hours before conducting FRAP on Zeiss 501 as described previously (Kedersha et al. 2005). Acquired FRAP images were converted to parametric 
data with the use of ImageJ software and ImageJ macro programming language (Rueden et al. 2017) .

503 Initially, image stacks were subjected to drift correction using Manual Drift Correction Plugin implemented

504 into the ImageJ macro source code (Manual drift correction (Fiji), Benoit Lombardot). Transformed

505 sequence image stacks were aligned with $\mathrm{ROI}$ of each bleached area for subsequent parametric data

506 acquisition. The output parametric data from each ROI was grouped into 3 categories, encompassing:

507 bleached, background, and reference region. Results were exported to csv files and subsequently

508 imported into R programming environment to facilitate calculations and plot generation (R Core Team

509 (2019). R: A language and environment for statistical computing. R Foundation for Statistical Computing,

510 Vienna, Austria (URL: https://www.R-project.org/). To eliminate noisy data Background Intensity Values

511 (BG) were subtracted from Bleach Intensity Values (B) to obtain Bleach Corrected Values (B_corr) for

512 each bleached region. Subsequently, Background Intensity Values were subtracted from Reference

513 Intensity Values (Ref) to obtain Reference Corrected Values (Ref_corr). Final calculation was based on

514 normalization of Bleach Corrected Values to Reference Corrected Values according to following equation:

515 Normalized Bleach Corrected Values = Bleach Corrected Values/ Reference Corrected Values. Final data

516 normalization, plots and total recovery summary tables were generated with the use of Frapplot package

517 (Guanqiao Ding (2019). frapplot: Automatic Data Processing and Visualization for FRAP. R package

518 version 0.1.3. URL: https://CRAN.R-project.org/package=frapplot).

\section{CRISPR/Cas9 Knockout of HRI}

521 Oligonucleotides encoding gRNAs targeting the first exon of HRI were designed using CRISPR Design 522 software from the Zhang lab (crispr.mit.edu). Oligonucleotide were annealed and cloned into pCas-Guide 523 (Origene) according to manufacturer's protocol. gRNA targeting the first exon of HRI contained the following 524 sequence: GCCCTCGGCGGGAAAGTCGA. pCas-guide plasmids were co-transfected with pDonor-D09 525 (GeneCopoeia), which carries a Puromycin resistance cassette, using Lipofectamine 2000 (Invitrogen). The 526 following day, cells were selected with $1.5 \mu \mathrm{g} / \mathrm{ml}$ of puromycin. Selection was allowed to continue for $24 \mathrm{hrs}$ 527 to lessen the likelihood of genomic incorporation of pDonor-D09. Cells were screened based on their failure 528 to form stress granules or phosphorylate elF2 $\alpha$ after exposure to $\mathrm{NaAsO}_{2}$. Cells were cloned by limiting 529 dilution. To confirm genomic ablation of HRI genomic DNA was purified as previously described(Kedersha 
et al. 2016). Cells were resuspended at 108 cells/ $\mathrm{mL}$ in digestion buffer $(100 \mathrm{mM} \mathrm{NaCl}, 10 \mathrm{mM}$ Tris $[\mathrm{pH}$ 8.0], $25 \mathrm{mM}$ EDTA [pH 8.0], 0.5\% SDS, $0.1 \mathrm{mg} / \mathrm{ml}$ proteinase $\mathrm{K}$ ) and incubated overnight at 55॰C. DNA

532 was extracted with phenol/chloroform and precipitated with $2.5 \mathrm{M}$ ammonium acetate and 2 volumes of

$533100 \%$ ethanol, washed with $70 \%$ ethanol and air dried. DNA pellet was resuspended in TE containing $0.1 \%$

534 SDS and RNase A $(1 \mu \mathrm{g} / \mathrm{ml})$ and incubated at 37॰C for 1 hour. DNA was extracted with phenol/chloroform

535 and precipitated as previously described. Resulting pellet was resuspended at a concentration of $100 \mathrm{ng} / \mu \mathrm{l}$.

536 For genotyping, the first exon of HRI was amplified by PCR using primers located within the promoter and

537 first intron (Forward: CTAGCTGCAGCATCGGAGT, Reverse: GAGGCAGACGTTCTTTTCAA) using

538 AccuPrime G-C rich polymerase (Invitrogen). Amplicons were cloned into pGEM-T Easy vector (Promega)

539 and sequenced.

540

\section{Data Availability}

542 The authors declare that there are no primary datasets and computer codes associated with this study.

\section{Acknowledgments}

545 We would like to thank Drs. Barbara Sollner-Webb and Denis Lafontaine for helpful comments before

546 submission of this manuscript. This work was funded by grants from the United States NIH (GM124458 to

547 SML, GM126150 to PI, GM126901 to PA) and National Science Centre in Poland (UMO$548 \quad 2015 / 17 / B / N Z 7 / 03043$ to WS).

\section{Author Contributions}

551 S.M.L. conceived, designed and preformed the analysis, interpreted results and took the lead in writing the

552 paper. W.S., M.S., M.L., S.O., A.A., preformed analysis, interpreted results and designed experiments. D.D.

553 and S.M. preformed analysis and aided in sample preparation. P.A. and P.I. gave critical feedback and 554 interpreted results.

555

556 


\section{REFERENCES}

Andersen JS, Lyon CE, Fox AH, Leung AK, Lam YW, Steen H, Mann M, Lamond Al. 2002. Directed proteomic analysis of the human nucleolus. Curr Biol 12: 1-11.

Audas TE, Audas DE, Jacob MD, Ho JJ, Khacho M, Wang M, Perera JK, Gardiner C, Bennett CA, Head T et al. 2016. Adaptation to Stressors by Systemic Protein Amyloidogenesis. Dev Cell 39: 155-168.

Audas TE, Jacob MD, Lee S. 2012. Immobilization of proteins in the nucleolus by ribosomal intergenic spacer non-coding RNA. Mol Cell 45: 147-157.

Aulas A, Fay MM, Lyons SM, Achorn CA, Kedersha N, Anderson P, Ivanov P. 2017. Stress-specific differences in assembly and composition of stress granules and related foci. J Cell Sci 130: 927-937.

Boulon S, Westman BJ, Hutten S, Boisvert FM, Lamond AI. 2010. The nucleolus under stress. Mol Cell 40: 216-227.

Brangwynne CP, Eckmann CR, Courson DS, Rybarska A, Hoege C, Gharakhani J, Julicher F, Hyman AA. 2009. Germline P granules are liquid droplets that localize by controlled dissolution/condensation. Science 324: 1729-1732.

Catez F, Dalla Venezia N, Marcel V, Zorbas C, Lafontaine DLJ, Diaz JJ. 2019. Ribosome biogenesis: An emerging druggable pathway for cancer therapeutics. Biochem Pharmacol 159: 74-81.

Chan PK, Aldrich M, Busch H. 1985. Alterations in immunolocalization of the phosphoprotein B23 in HeLa cells during serum starvation. Exp Cell Res 161: 101-110.

Damgaard CK, Lykke-Andersen J. 2011. Translational coregulation of 5'TOP mRNAs by TIA-1 and TIAR. Genes Dev 25: 2057-2068.

Dundr M, Misteli T. 2010. Biogenesis of nuclear bodies. Cold Spring Harb Perspect Biol 2: a000711.

Emara MM, Fujimura K, Sciaranghella D, Ivanova V, Ivanov P, Anderson P. 2012. Hydrogen peroxide induces stress granule formation independent of elF2alpha phosphorylation. Biochem Biophys Res Commun 423: 763-769.

Feric M, Vaidya N, Harmon TS, Mitrea DM, Zhu L, Richardson TM, Kriwacki RW, Pappu RV, Brangwynne CP. 2016. Coexisting Liquid Phases Underlie Nucleolar Subcompartments. Cell 165: 1686-1697.

Grummt I, Grummt F. 1976. Control of nucleolar RNA synthesis by the intracellular pool sizes of ATP and GTP. Cell 7: 447-453.

Ivanov P, Kedersha N, Anderson P. 2011. Stress puts TIA on TOP. Genes Dev 25: 2119-2124.

Kedersha N, Ivanov P, Anderson P. 2013. Stress granules and cell signaling: more than just a passing phase? Trends Biochem Sci 38: 494-506.

Kedersha N, Panas MD, Achorn CA, Lyons S, Tisdale S, Hickman T, Thomas M, Lieberman J, McInerney GM, Ivanov P et al. 2016. G3BP-Caprin1-USP10 complexes mediate stress granule condensation and associate with 4OS subunits. J Cell Biol 212: 845-860.

Kedersha N, Stoecklin G, Ayodele M, Yacono P, Lykke-Andersen J, Fitzler M, Scheuner D, Kaufman R, Golan DE, Anderson P. 2005. Stress granules and processing bodies are dynamically liked sites of mRNP remodeling. J Cell Biol 169: 871-884.

Kos-Braun IC, Jung I, Kos M. 2017. Tor1 and CK2 kinases control a switch between alternative ribosome biogenesis pathways in a growth-dependent manner. PLoS Biol 15: e2000245. 
Lin Y, Protter DS, Rosen MK, Parker R. 2015. Formation and Maturation of Phase-Separated Liquid Droplets by RNA-Binding Proteins. Mol Cell 60: 208-219.

Liu Y, Chen JJ, Gao Q, Dalal S, Hong Y, Mansur CP, Band V, Androphy EJ. 1999. Multiple functions of human papillomavirus type 16 E6 contribute to the immortalization of mammary epithelial cells. J Virol 73: 7297-7307.

Liu Y, Liang S, Tartakoff AM. 1996. Heat shock disassembles the nucleolus and inhibits nuclear protein import and poly(A)+ RNA export. EMBO J 15: 6750-6757.

Lyons SM, Achorn C, Kedersha NL, Anderson PJ, Ivanov P. 2016. YB-1 regulates tiRNA-induced Stress Granule formation but not translational repression. Nucleic Acids Res 44: 69496960.

Lyons SM, Anderson P. 2016. RNA-Seeded Functional Amyloids Balance Growth and Survival. Dev Cell 39: 131-132.

Martin RM, Ter-Avetisyan G, Herce HD, Ludwig AK, Lattig-Tunnemann G, Cardoso MC. 2015. Principles of protein targeting to the nucleolus. Nucleus 6: 314-325.

Mayer C, Bierhoff H, Grummt I. 2005. The nucleolus as a stress sensor: JNK2 inactivates the transcription factor TIF-IA and down-regulates rRNA synthesis. Genes Dev 19: 933-941.

Mayer C, Zhao J, Yuan X, Grummt I. 2004. mTOR-dependent activation of the transcription factor TIF-IA links rRNA synthesis to nutrient availability. Genes Dev 18: 423-434.

Meyuhas O, Kahan T. 2015. The race to decipher the top secrets of TOP mRNAs. Biochim Biophys Acta 1849: 801-811.

Mitrea DM, Cika JA, Guy CS, Ban D, Banerjee PR, Stanley CB, Nourse A, Deniz AA, Kriwacki RW. 2016. Nucleophosmin integrates within the nucleolus via multi-modal interactions with proteins displaying R-rich linear motifs and rRNA. Elife 5.

Mitrea DM, Cika JA, Stanley CB, Nourse A, Onuchic PL, Banerjee PR, Phillips AH, Park CG, Deniz AA, Kriwacki RW. 2018. Self-interaction of NPM1 modulates multiple mechanisms of liquid-liquid phase separation. Nat Commun 9: 842.

Montanaro L, Trere D, Derenzini M. 2008. Nucleolus, ribosomes, and cancer. Am J Pathol 173: 301-310.

Mullineux ST, Lafontaine DL. 2012. Mapping the cleavage sites on mammalian pre-rRNAs: where do we stand? Biochimie 94: 1521-1532.

Novoa I, Zhang Y, Zeng H, Jungreis R, Harding HP, Ron D. 2003. Stress-induced gene expression requires programmed recovery from translational repression. EMBO J 22: 1180-1187.

Pestov DG, Lapik YR, Lau LF. 2008. Assays for ribosomal RNA processing and ribosome assembly. Curr Protoc Cell Biol Chapter 22: Unit 2211.

Protter DSW, Rao BS, Van Treeck B, Lin Y, Mizoue L, Rosen MK, Parker R. 2018. Intrinsically Disordered Regions Can Contribute Promiscuous Interactions to RNP Granule Assembly. Cell Rep 22: 1401-1412.

Rubbi CP, Milner J. 2003. Disruption of the nucleolus mediates stabilization of p53 in response to DNA damage and other stresses. EMBO J 22: 6068-6077.

Rueden CT, Schindelin J, Hiner MC, DeZonia BE, Walter AE, Arena ET, Eliceiri KW. 2017. ImageJ2: Image for the next generation of scientific image data. BMC Bioinformatics 18: 529.

Schneider DA, Michel A, Sikes ML, Vu L, Dodd JA, Salgia S, Osheim YN, Beyer AL, Nomura M. 2007. Transcription elongation by RNA polymerase I is linked to efficient rRNA processing and ribosome assembly. Mol Cell 26: 217-229. 
Szaflarski W, Fay MM, Kedersha N, Zabel M, Anderson P, Ivanov P. 2016. Vinca alkaloid drugs promote stress-induced translational repression and stress granule formation. Oncotarget 7: 30307-30322.

Tafforeau L, Zorbas C, Langhendries JL, Mullineux ST, Stamatopoulou V, Mullier R, Wacheul L, Lafontaine DL. 2013. The complexity of human ribosome biogenesis revealed by systematic nucleolar screening of Pre-rRNA processing factors. Mol Cell 51: 539-551.

Tatomer DC, Terzo E, Curry KP, Salzler H, Sabath I, Zapotoczny G, McKay DJ, Dominski Z, Marzluff WF, Duronio RJ. 2016. Concentrating pre-mRNA processing factors in the histone locus body facilitates efficient histone mRNA biogenesis. J Cell Biol 213: 557-570.

Thoreen CC, Chantranupong L, Keys HR, Wang T, Gray NS, Sabatini DM. 2012. A unifying model for mTORC1-mediated regulation of mRNA translation. Nature 485: 109-113.

Van Treeck B, Parker R. 2018. Emerging Roles for Intermolecular RNA-RNA Interactions in RNP Assemblies. Cell 174: 791-802.

Van Treeck B, Protter DSW, Matheny T, Khong A, Link CD, Parker R. 2018. RNA self-assembly contributes to stress granule formation and defining the stress granule transcriptome. Proc Natl Acad Sci U S A 115: 2734-2739.

Warner JR, Vilardell J, Sohn JH. 2001. Economics of ribosome biosynthesis. Cold Spring Harb Symp Quant Biol 66: 567-574.

Woods SJ, Hannan KM, Pearson RB, Hannan RD. 2015. The nucleolus as a fundamental regulator of the $\mathrm{p} 53$ response and a new target for cancer therapy. Biochim Biophys Acta 1849: 821-829.

Xu H, Pillai RS, Azzouz TN, Shpargel KB, Kambach C, Hebert MD, Schumperli D, Matera AG. 2005. The C-terminal domain of coilin interacts with $\mathrm{Sm}$ proteins and U snRNPs. Chromosoma 114: 155-166.

Yang K, Yang J, Yi J. 2018. Nucleolar Stress: hallmarks, sensing mechanism and diseases. Cell Stress 2: 125-140.

Yao RW, Xu G, Wang Y, Shan L, Luan PF, Wang Y, Wu M, Yang LZ, Xing YH, Yang L et al. 2019. Nascent Pre-rRNA Sorting via Phase Separation Drives the Assembly of Dense Fibrillar Components in the Human Nucleolus. Mol Cell 76: 767-783 e711.

Zatsepina OV, Voronkova LN, Sakharov VN, Chentsov YS. 1989. Ultrastructural changes in nucleoli and fibrillar centers under the effect of local ultraviolet microbeam irradiation of interphase culture cells. Exp Cell Res 181: 94-104.

\section{Figure 1. rRNA processing is inhibited in response to ISR-activating stress.}

(A) Schematic of 5-EU metabolic labeling experiment. Cells were stressed for $1.5 \mathrm{hrs}$ and then 5-EU was added for 30 minutes to monitor transcriptional output. (B) Transcription in the nucleolus has been inhibited after 2 hours of $\mathrm{NaAsO}_{2}$-induced stress as indicated by loss of 5-EU signal in the nucleolus. (C) Schematic of the maturation pathway of human rRNAs. Approximate locations of northern blotting probes are noted in magenta, green and blue. (D) Northern blotting of cellular RNA after indicated treatments. 5 
$\mu \mathrm{g}$ of RNA was resolved on $1.2 \%$ HEPES-EDTA agarose gel, transferred to nylon membrane and probed

687 with indicated northern blotting probes. $\mathrm{NaAsO}_{2}$ and lomustine result in accumulation of $47 \mathrm{~S}$ pre-rRNA,

688 the generation of the aberrant 34S product and the decrease in downstream precursors (18S-E, 21S,

$68926 \mathrm{~S}, 41 \mathrm{~S}, 12 \mathrm{~S}$ ). (E) qRT-PCR confirms increase in 47S pre-rRNA precursor after $\mathrm{NaAsO}_{2}$ and Lomustine

690 treatment and the decrease in 47S levels after ActD treatment. Data analyzed by student t-test ${ }^{* *}=$

$\left.691 p<0.01,{ }^{* * * *} p<0.0001\right)$.

692

Figure 2. Stalled rRNA processing intermediates are utilized following a return to homeostasis.

(A) Schematic of [32P]-ortho-phosphoric acid labeling experiment. Cells were stressed for 10 minutes

before addition of $\left[{ }_{32} \mathrm{P}\right]$ to monitor rRNA transcription $(\mathrm{B}) \mathrm{NaAsO}_{2}$-treatment results in inhibition of $r$ RNA processing. Large precursor rRNAs are generated, but the never mature into $18 \mathrm{~S}$ and $28 \mathrm{~S}$ rRNAs. (C) End-point metabolic labeling with cells treated with $\mathrm{NaAsO}_{2}$ or Actinomycin $\mathrm{D}$ (ActD). Whereas $\mathrm{NaAsO}_{2}$ inhibits rRNA processing, ActD prevents rRNA transcription. (D) Schematic of [32P]-ortho-phosphoric acid labeling recovery experiment. Cells were stressed and labeled as in (A), but following 2 hours, $\mathrm{NaAsO}_{2}$ was washed out and cells were allowed to recover over the indicated time course. (E) After $\mathrm{NaAsO}_{2}$ washout, rRNA that was labeled during the initial phases of stress response re-entered the rRNA maturation pathway resulting in mature $18 \mathrm{~S}$ and $28 \mathrm{~S}$ rRNAs. Treatment with ActD prevents labeling of rRNA at the beginning of the time-course $(120 \mathrm{~m})$ or after washout $(660 \mathrm{~m})$.

Figure 3. Nucleolar structure is maintained upon activation of the Integrated Stress Response.

707 (A) Immunofluorescence of untreated U2OS osteosarcoma cells (Aa) or treated with Actinomycin D (Ab),

$708 \mathrm{NaAsO}_{2}(\mathbf{A c})$, Lomustine $\mathrm{H}_{2} \mathrm{O}_{2}(\mathbf{A d})$, or $\mathrm{H}_{2} \mathrm{O}_{2}(\mathbf{A e})$. Nucleolar integrity was monitored by staining for 709 nucleophosmin (NPM, green) and activation of the ISR was monitored by analysis of stress granule

710 formation using elF3B (red). (B) Nucleolar number is unaffected by stress response on a per cell basis.

711 Cells were treated with indicated stresses and the number of retained nucleoli per nuclei were manually

712 counted using NPM as a nucleolar marker. (C) Representative images of Imaris 3D reconstruction after

713 indicated treatments. (D) Nucleolar volume is unaffected after ISR-activating stresses ( $\mathrm{NaAsO}_{2}$ and

714 Lomustine), while volume is significantly decreased after mTOR-inactivating $\mathrm{H}_{2} \mathrm{O}_{2}$ treatment and ActD, 
715 which inhibits transcription. Data analyzed by student t-test $\left({ }^{*}=p<0.05,{ }^{* * * *} p<0.0001\right)$. (E) Sphericity of

716 nucleoli was determined following 3D reconstruction. ISR-activating stresses results in a more spherical

717 nucleolar structure, while mTOR-inactivating $\mathrm{H}_{2} \mathrm{O}_{2}$ treatments results in a statistically significant decrease

718 in sphericity Data analyzed by student t-test $\left({ }^{*}=p<0.05,{ }^{* * *} p<0.0001\right)$.

721 Figure 4. Unprocessed rRNA is stored in the nucleolus resulting in perturbed nucleolar dynamics.

722 (A) 3D reconstructions of nucleoli after indicated treatment using NPM and FISH probes to 5'ETS.

723 Results demonstrate that unprocessed rRNA is retained within the nucleolus during a stress response. (B

724 - E) Fluorescence recovery after photobleaching of RPL7A (C - C) or Nol9 (D - E) under basal and

725 stressed conditions. Under nominal conditions, the bleached fluorescence signal rapidly recovers as

726 expected by an active organelle. However, fluorescence recovery is severely perturbed during a stress

727 response, consistent with the inhibition of rRNA processing. Red arrowheads denote the photobleached

728 nucleoli.

732 (A) 5-EU metabolic labeling was done as before with $0 \mu \mathrm{M}(\mathbf{A a}), 75 \mu \mathrm{M}$ (Ab) or $200 \mu \mathrm{M} \mathrm{NaAsO} 2$ (Ac).

733 Formation of stress granules, as monitored by elF3B, was used as a proxy for activation of the ISR. rRNA

734 transcription was impaired after 2 hours of stress regardless of the formation of stress granules. Red

735 arrowheads denote the nucleoli and green arrowheads denote stress granules. (B) 5-EU metabolic of WT

736 (Ba-b) or $\Delta \mathrm{HRI}(\mathrm{Bc}-\mathrm{d})$ cells untreated or treated with $\mathrm{NaAsO}_{2} . \Delta \mathrm{HRI}$ cells fail to form stress granules as

737 translation is not regulated in these cells in response to stress. However, rRNA transcription is still

738 inhibited 2 hours post-stress induction. (C) Northern blotting of rRNA in WT and $\triangle \mathrm{HRI}$ U2OS cells. Both

739 cells respond to $\mathrm{NaAsO}_{2}$ by generating the $34 \mathrm{~S}$ fragment, diagnostic of processing inhibition (In 9 \& 12)

740 and leading to the reduction of downstream precursors (In 15, 18, 21, \& 24). (D) [32P]-metabolic labeling

741 of WT and $\Delta \mathrm{HRI}$ cells demonstrate an inhibition in rRNA processing in response to cell stress despite a

742 failure to inhibit translation. 


\section{Figure 6. Failure to regulate rRNA processing with translation inhibition results in nucleolar}

\section{5 fragmentation.}

746 (A - D) FRAP of U2OS cells stably expressing RPL7A (A - B) or Nol9 (C - D) untreated or treated with

747 cycloheximide or puromycin to repress translation without inducing a stress response. Despite inhibition

748 of translation, nucleolar dynamics remain unaltered indicating that previously identified alteration in

749 nucleolar dynamics is not a result of translation inhibition. Red arrowheads denote photobleached

750 nucleoli. (E) Northern blotting of rRNA after treatment with $\mathrm{CHX}$ and puro demonstrates that 47S rRNA

751 and 34S fragment do not accumulate. Instead, there is a reduction in 47S levels (In 9 \& 10)

752 commensurate with the inhibition of ribosomal protein synthesis. (F) After indicated treatments, nucleoli of

753 U2OS cells were analyzed by immunofluorescence by staining for NPM. Stress granule formation was

754 monitored by elF3B localization. As shown previously, $\mathrm{NaAsO}_{2}$ results in stress granule formation and

755 maintenance of nucleolar structure. However, translational inhibitors that do not trigger a stress response

756 (CHX and Puro) result in fragmentation of nucleoli.

\section{Supplemental Figure 1.}

759 (A) Response of $\mathrm{U} 2 \mathrm{OS}$ cells to indicated treatments. $\mathrm{NaAsO}_{2}$, Lomustine and $\mathrm{H}_{2} \mathrm{O}_{2}$ activate the ISR as

760 indicated by phosphorylation of elF2 $\alpha$, while $\mathrm{H}_{2} \mathrm{O}_{2}$ also deactivated mTOR as indicated by

761 dephosphorylation of 4EBP1. CHX and puromycin affect neither pathway. (B) p-53 null HeLa cells were

762 treated with indicated stressors and rRNA was analyzed by northern blotting. rRNA processing was

763 altered in a similar manner as in p53-positive U2OS cells. (C) Untransformed RPE-1 cells were treated

764 with indicated stressors and rRNA was analyzed by northern blotting. rRNA processing was altered in a

765 similar manner as to cancerous U2OS and HeLa cells. (D) Mouse NIH3T3 cells were treated with

766 indicated stressors and rRNA was analyzed by northern blotting. rRNA processing was altered in a similar

767 manner as to human U2OS, HeLa and RPE-1 cells. (E) Generation of 34S fragment and depletion of

768 downstream precursors occurs in a time- and dose-dependent manner in U2OS cells. 


\section{Supplemental Figure 2.}

$772(\mathbf{A}-\mathbf{H})$ U2OS cells were treated with $\mathrm{NaAsO}_{2}$ and nucleolar markers were used to monitor nucleolar

773 integrity. PHF6 (A), RPA194 (B), BMS1 (C), elF6, TIF1A (D), FBL, NOP16 (E), DKC1 (F), CIRH1A (G),

774 and Ki67 (H) were analyzed. This suite of proteins contains representatives of each 3 nucleolar

775 subcompartments. (I) Nucleolar integrity is maintained in p53-deficient HeLa cells as monitored by NPM

776 staining.

777

778 Supplemental Figure 3.

779 (A) Generation of stable cell lines expressing mCherry-NPM, mCherry-Nol9 or EGFP-RPL7A. U2OS cells 780 were transfected with plasmids encoding tagged versions of indicated proteins, drug selected and single 781 cell clones were obtained by limiting dilution. Clones expressing near endogenous levels were selected

782 for future use. Tagged versions are revealed by slower mobility (B - C) FRAP of mCherry-NPM reveals

783 decreased mobility of this protein in response to stress in line with inhibition of rRNA processing.

785 Supplemental Figure 4.

786 (A) Genotyping of HRI locus reveals a 40-nucleotide deletion within the $1_{\text {st }}$ exon of HRI. This results in a 787 premature termination codon in the 2 nd exon leaving 13 exon junctions between the PTC and the natural 788 termination codon. Red lettering indicates location of gRNA. (B - C) Comparison of HRI protein sequence 789 from WT U2OS and hypothetical translation product from $\Delta \mathrm{HRI}$ cells. Green lettering indicates differences 790 downstream of frame shifting mutation. (D) U2OS $\Delta \mathrm{HRI}$ fail to form stress granules in response to $791 \mathrm{NaAsO}_{2}$, but still form stress granules in response to thapsigargin, which activates PERK. Cells were 792 treated with $100 \mu \mathrm{M} \mathrm{NaAsO}$ or $2 \mathrm{mM}$ thapsigargin for 1 hour and then prepared for immunofluorescence.

793 Cells were stained with DAPI (blue), elF4G (Green) and elF3B (Red). (E) $\Delta$ HRI cells do not inhibit

794 translation in response to $\mathrm{NaAsO}_{2}$, but still respond to thapsigargin. U2OS (WT) cells cease translation

795 upon treatment with $\mathrm{NaAsO}_{2}$ or thapsigargin. However, U2OS $\Delta \mathrm{HRI}$ cells are refractory to $\mathrm{NaAsO}_{2}-$

796 mediated translation inhibition, but maintain their response to thapsigargin. (F) Growth curves of U2OS 797 cells and U2OS $\Delta \mathrm{HRI}$ cells demonstrate no statistically significant difference in growth rates between the 798 two cell lines. 
A

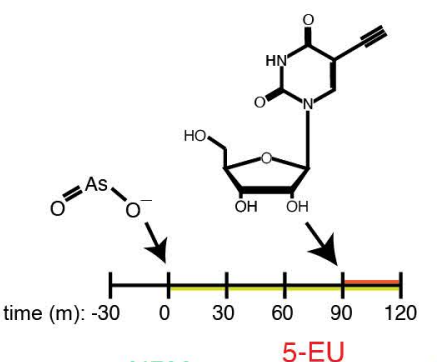

B

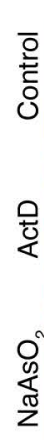

NPM (Nascent RNA

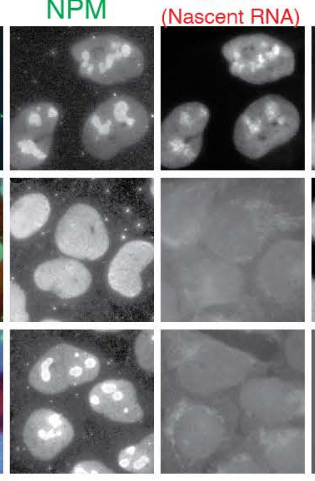

D

$\frac{\text { EtBr }}{\overline{0} \text { O }^{N}}$

$28 \mathrm{~s}$ ed

$18 \mathrm{~s}\|\mathrm{H}\| \mathrm{H}$

$\begin{array}{llll}1 & 2 & 3 & 4\end{array}$

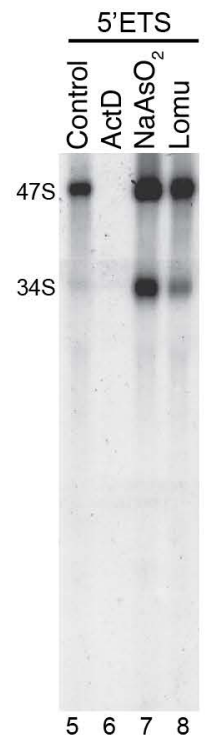

$\begin{array}{llll}5 & 6 & 7 & 8\end{array}$
$47 \mathrm{~S} / 45 \mathrm{~S}$

$26 \mathrm{~S}$

$21 S$

18S-E

26S

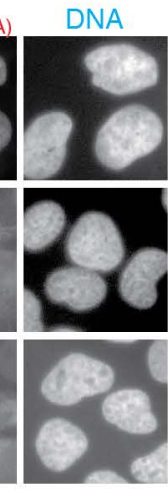

C

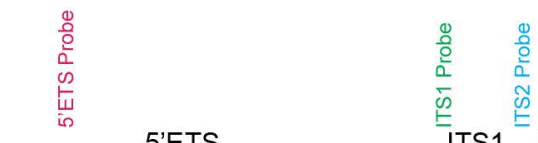
ITS1 ITS2
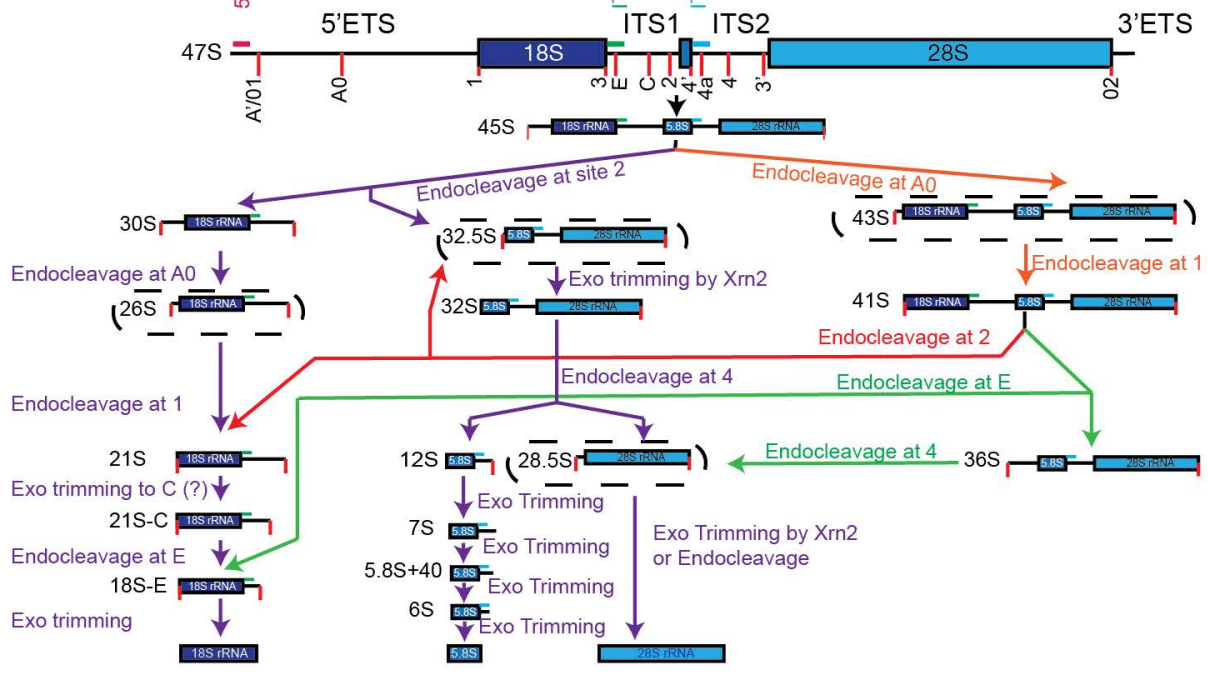

E
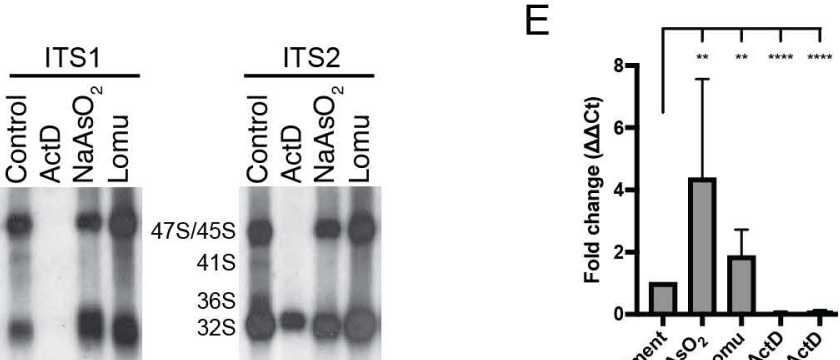

$12 S$

$41 \mathrm{~S}$

$36 \mathrm{~S}$

$32 \mathrm{~S}$

揵要雪

.

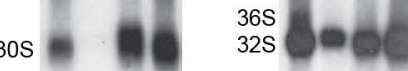

$9 \quad 101112$

13141516 
Figure 2

A

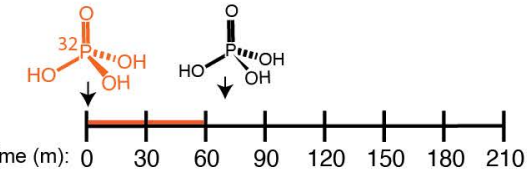

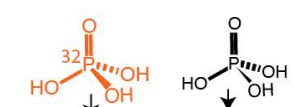

time $(m)$ :

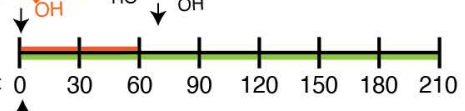

$0^{A S}$

D

Control

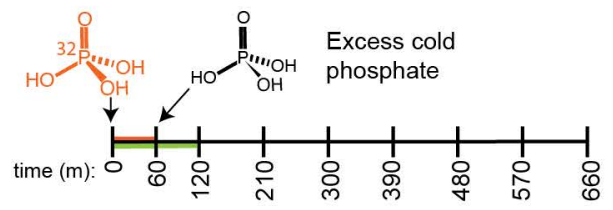

Arsenite Treated

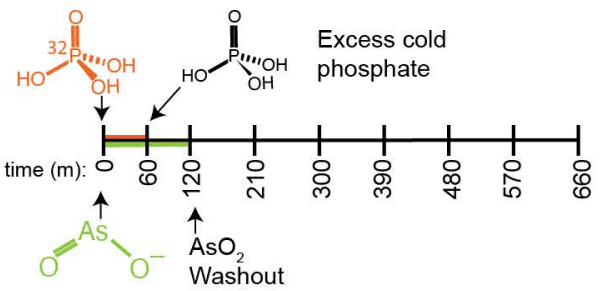

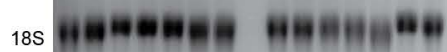

B

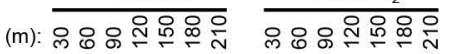

$28 \mathrm{~S}$

185

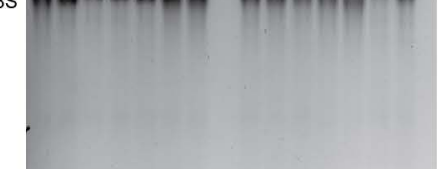

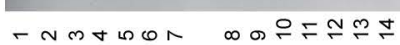

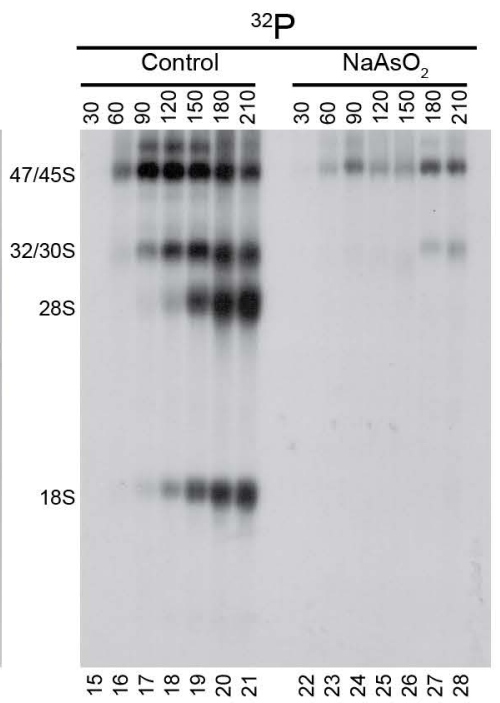

C

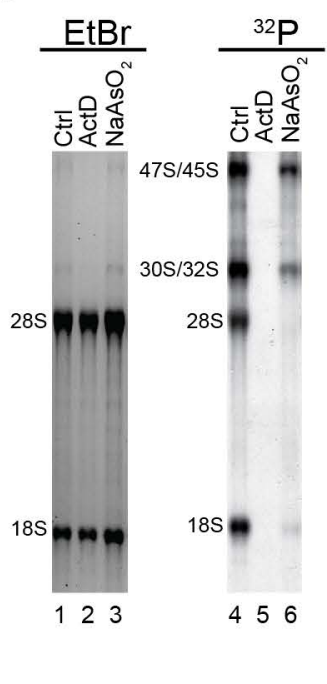

E

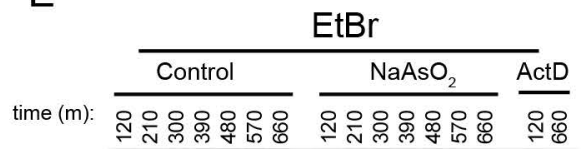

$28 \mathrm{~S}$

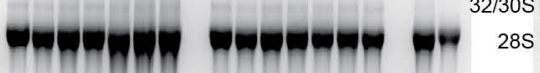

$18 \mathrm{~S}$

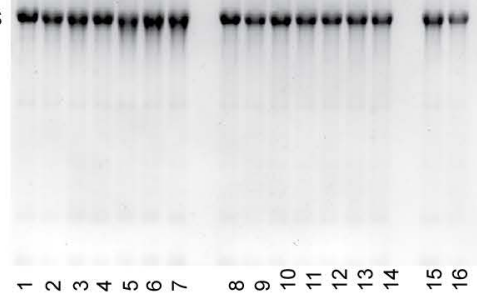

我

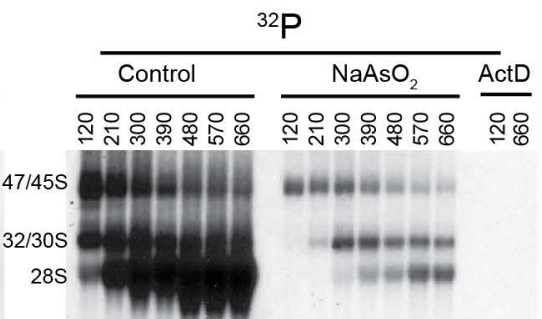

${ }^{32} \mathrm{P}$

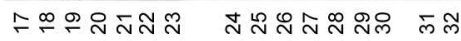


Figure 3

A
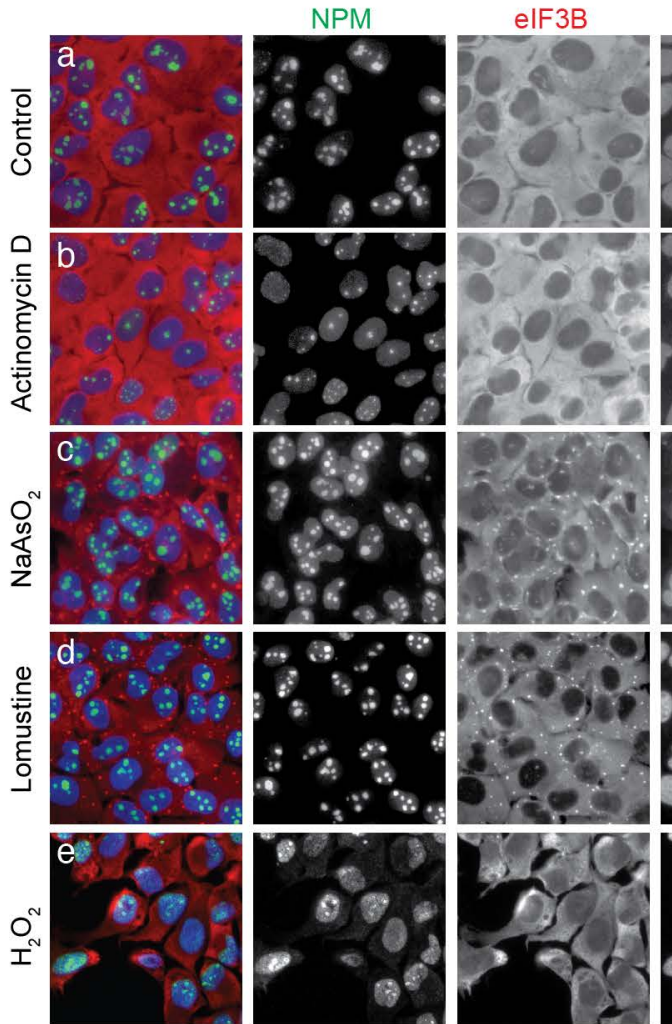

DNA

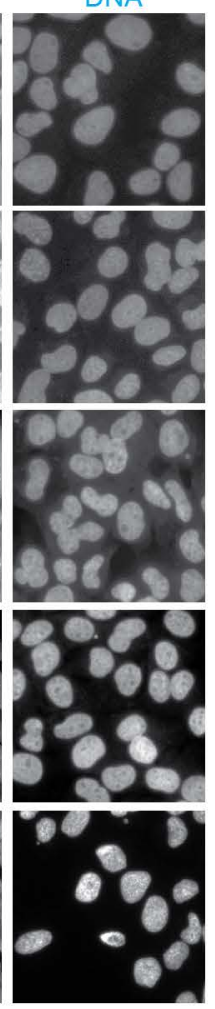

B

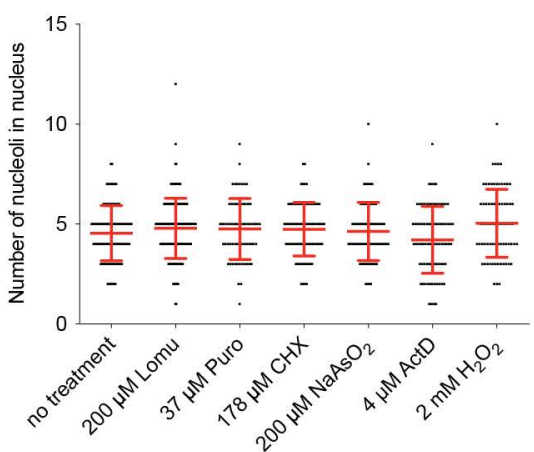

C
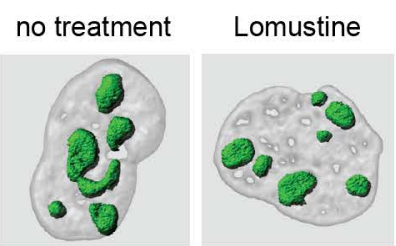

$\mathrm{NaAsO}_{2}$

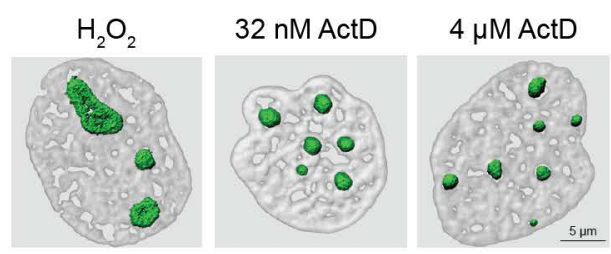

D

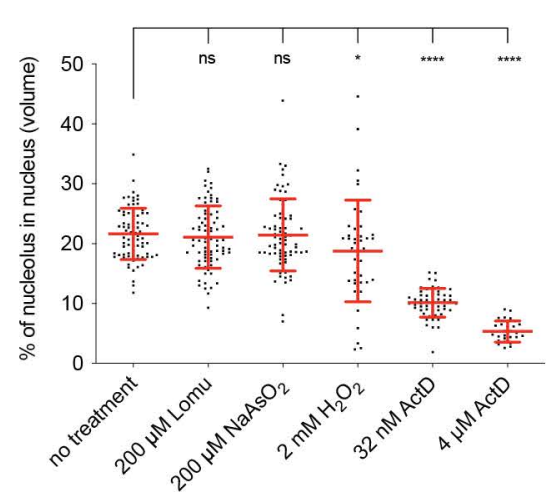

E

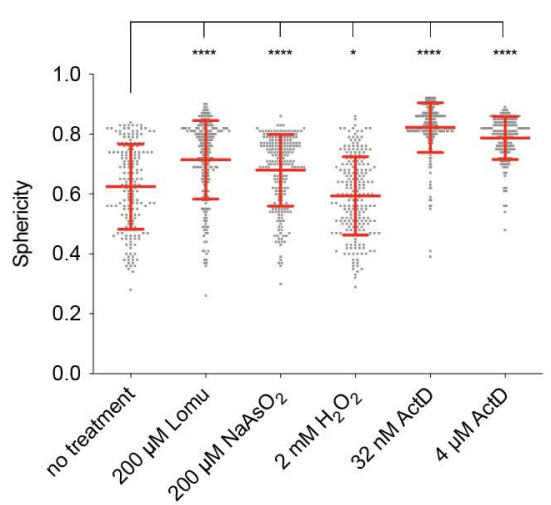


B

\begin{tabular}{|c|c|c|c|c|c|c|c|c|c|c|c|}
\hline & 0 & 1 & 5 & 10 & 15 & 20 & 25 & 30 & 35 & 40 & 45 \\
\hline \multirow{2}{*}{ 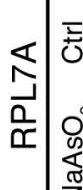 } & & 6 & $?$ & $?$ & ? & ? & $?$ & $?$ & 8 & $?$ & 8 \\
\hline & & & 88 & & 2 & 8 & 8 & \& & 8 & 8 & 8 \\
\hline
\end{tabular}

D

\section{C}
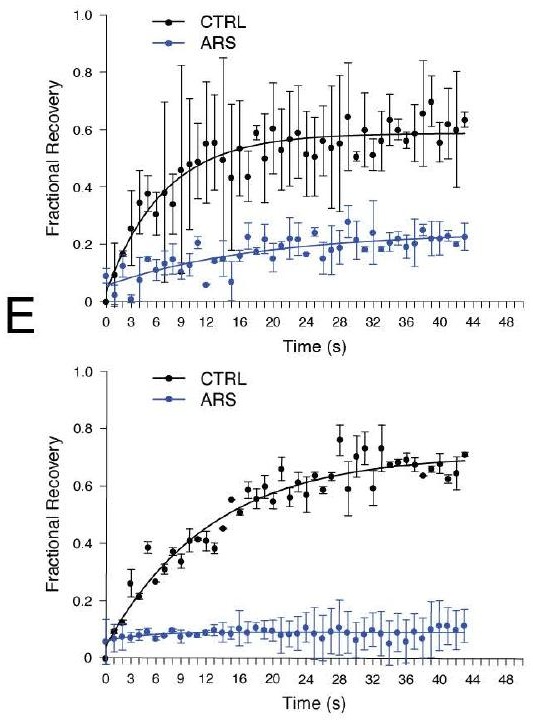

\section{C} Time (s)
A

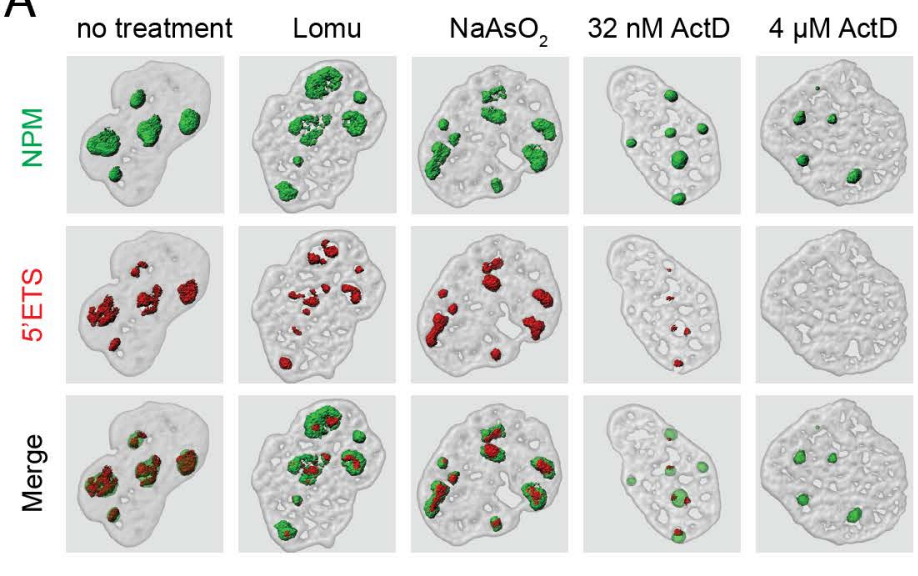

Figure 4

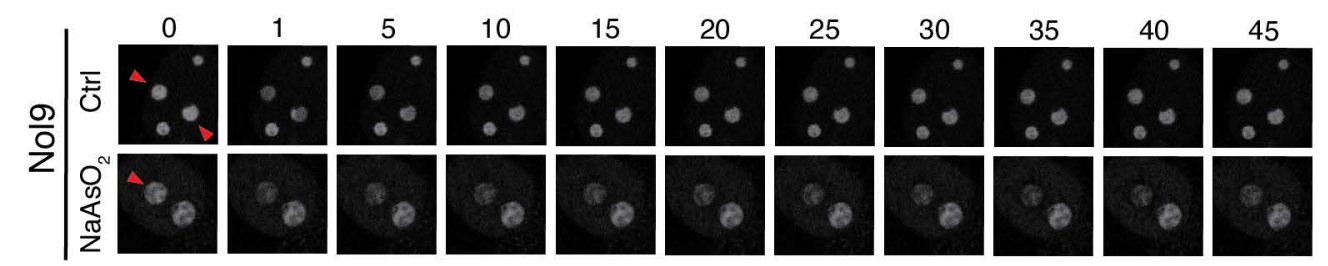

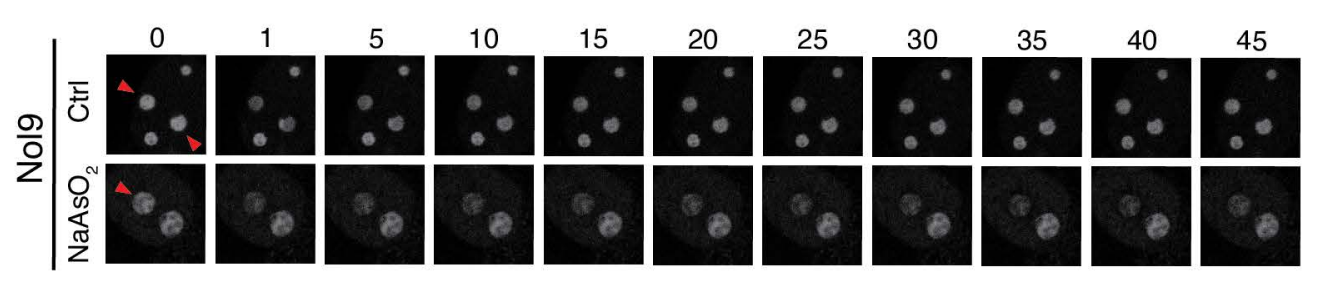


Figure 5
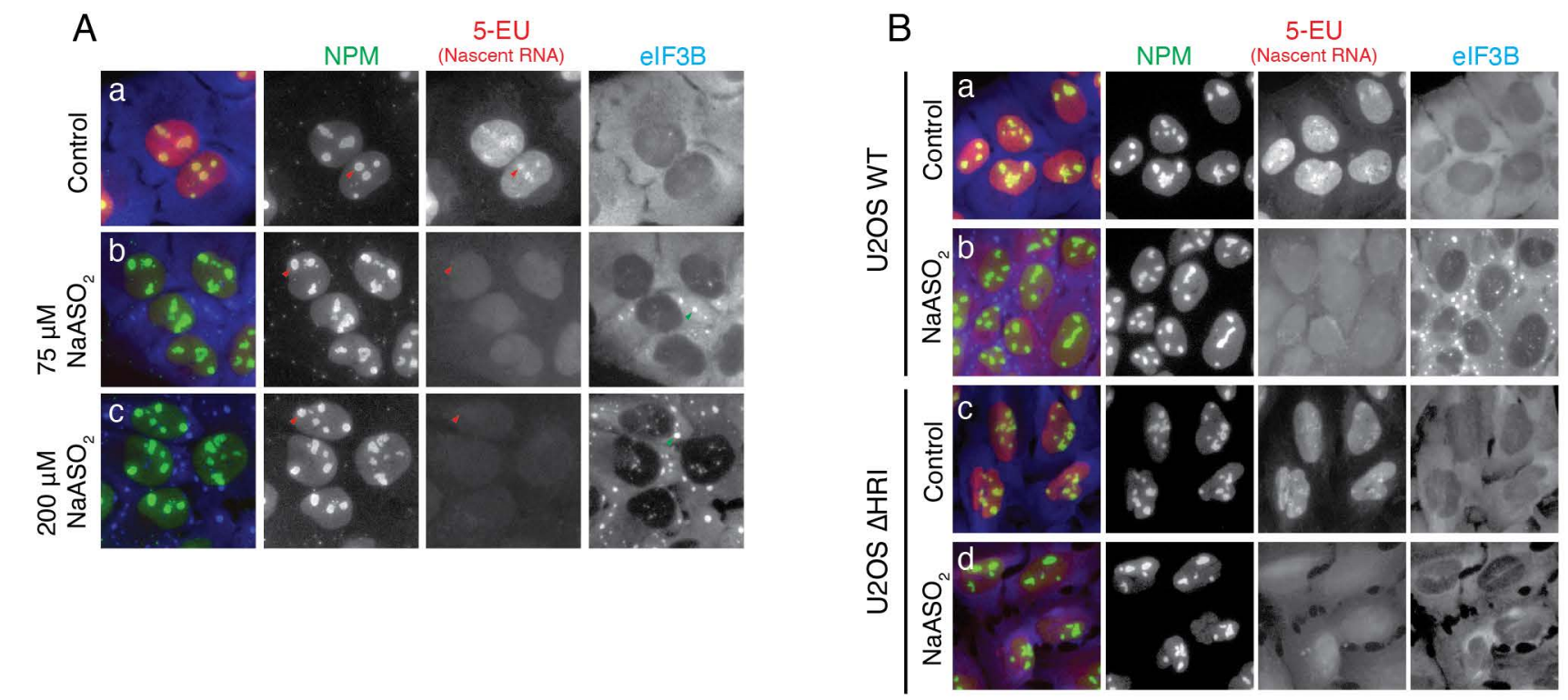

C

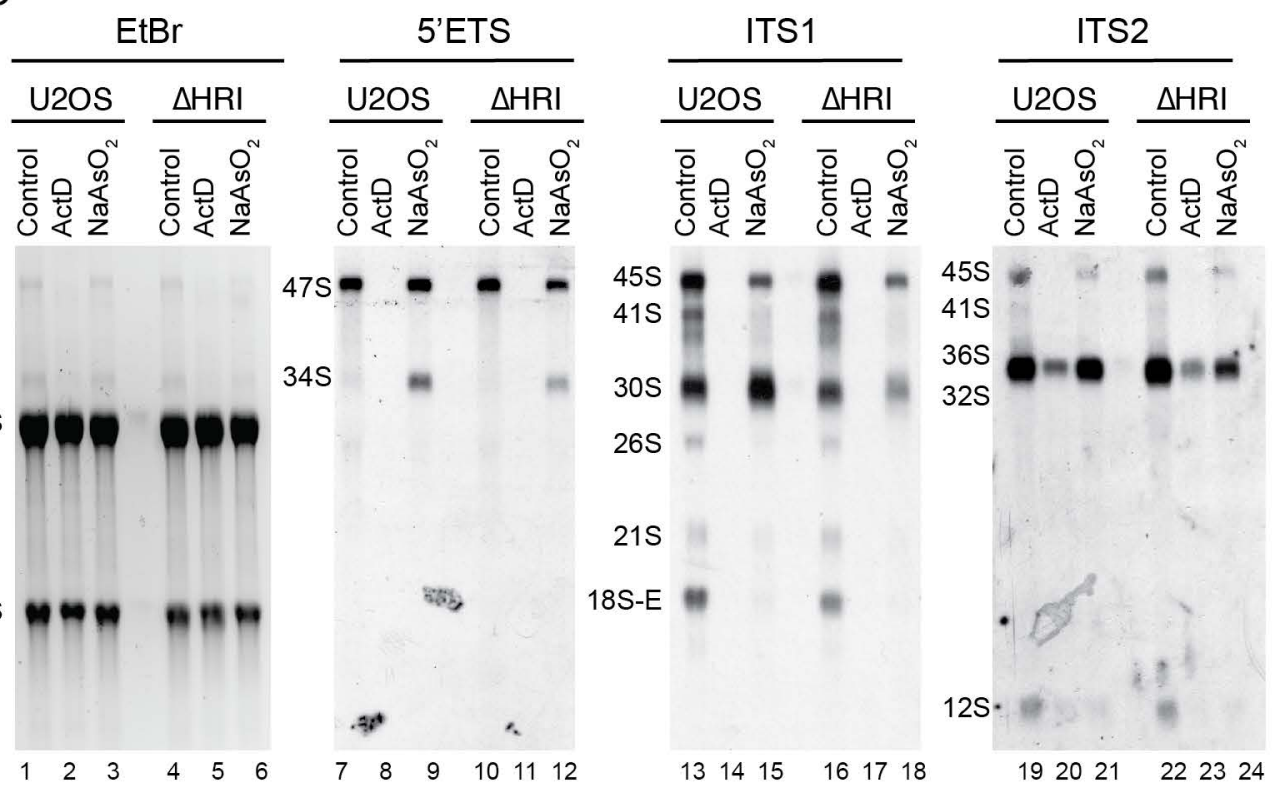

D

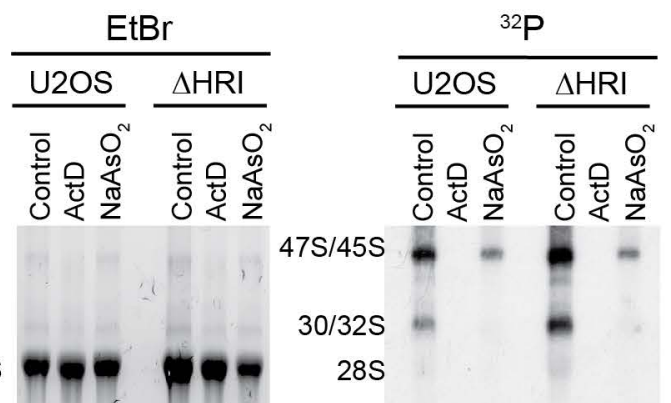

$18 \mathrm{~S} * \boldsymbol{m}$ 
Figure 6
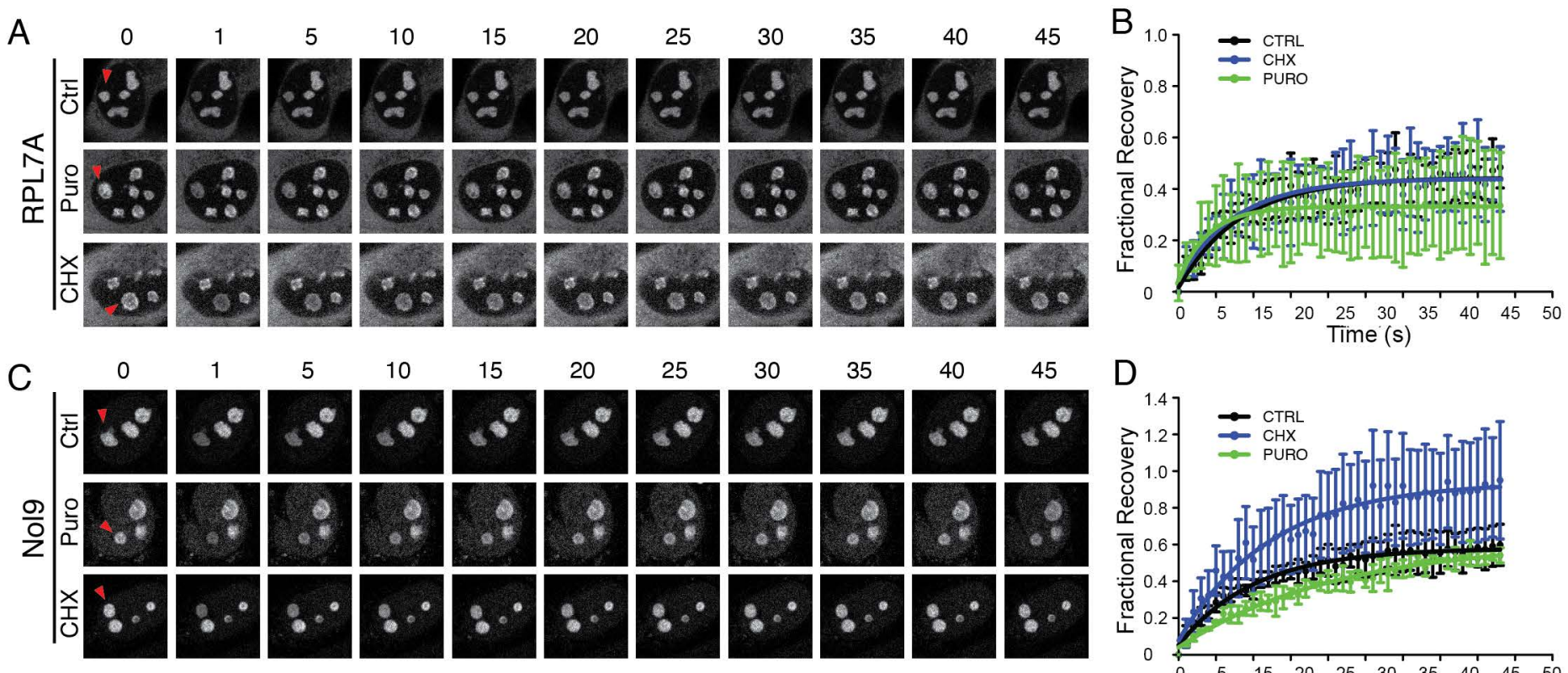

E

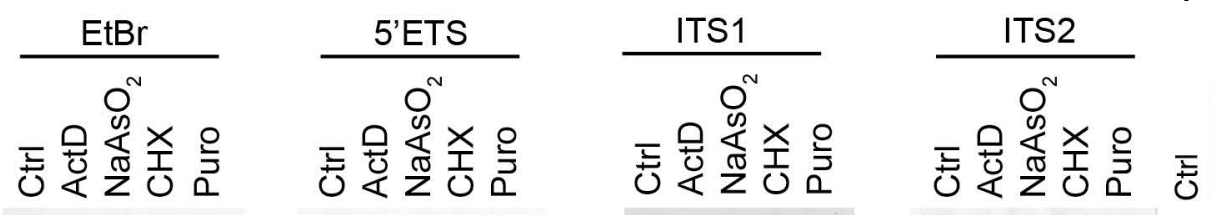

anda

\section{$34 S$ \\ 475}

m

$45 S$

$41 S$

$305=-6$

$26 S$

$21 S$

18S-E

$45 S=2-=$ $41 \mathrm{~S}$

$36 \mathrm{~S}$ $32 S$

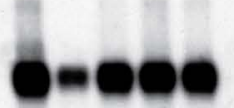

$12 S$
F

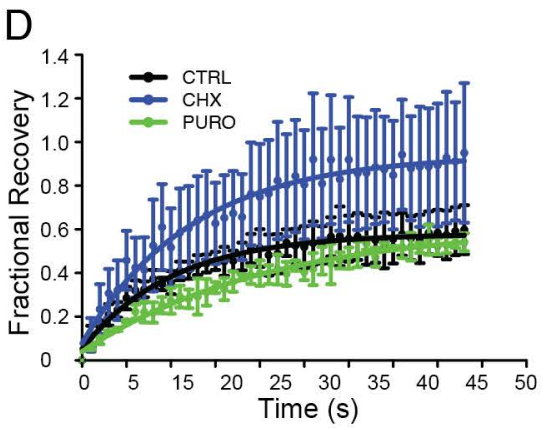

NPM

eIF3B
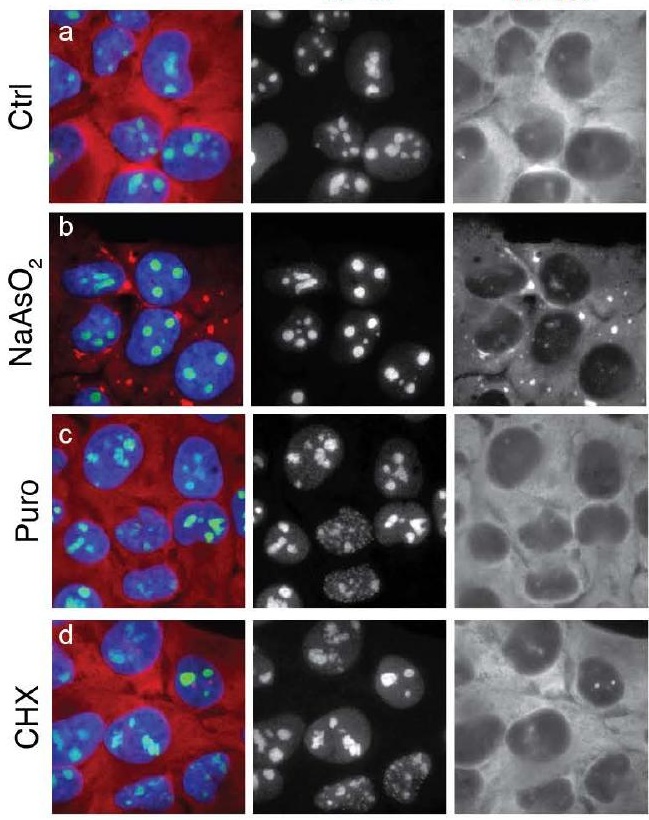